\title{
An updated catalogue of giant radio sources
}

\author{
A. Kuźmicz ${ }^{1,2,3 \star}$ M. Jamrozy, ${ }^{2}$ K. Bronarska K. Janda-Boczar ${ }^{2}$ D. J. Saikiaa ${ }^{4,5,6}$ \\ ${ }^{1}$ Center for Theoretical Physics, Polish Academy of Sciences, Al. Lotników 32/46, 02-668 Warsaw, Poland \\ ${ }^{2}$ Astronomical Observatory, Jagiellonian University, ul. Orla 171, 30-244 Krakow, Poland \\ ${ }^{3}$ Queen Jadwiga Astronomical Observatory in Rzepiennik Biskupi, 33-163 Rzepiennik Strzyżewski, Poland \\ ${ }^{4}$ Inter-University Centre for Astronomy and Astrophysics (IUCAA), Ganeshkhind, Pune 411 007, India \\ ${ }^{5}$ NCRA, TIFR, Post Bag 3, Ganeshkhind, Pune 411 007, India \\ ${ }^{6}$ Cotton University, Panbazar, Guwahati 781 001, India
}

Accepted August 10, 2018

\begin{abstract}
We present a catalogue of 349 giant radio sources (GRSs including both galaxies and quasars). The database contains all giants known to date from the literature. These GRSs cover the redshift range of $0.016<z<3.22$ and include radio sources of projected linear sizes larger than $0.7 \mathrm{Mpc}$ which extend up to $4.7 \mathrm{Mpc}$. We provide the principal parameters (i.e. exact position of the host in the sky, redshift, angular and projected linear size, red optical magnitude, radio morphology type, total radio flux density and luminosity) for all the sources as well as characteristics of the sample. Based on the distribution of GRSs in the sky we identify regions where there is a paucity of giants, so that future surveys for this type of objects could concentrate primarily in these fields. From the analysis presented here, we estimate a lower limit for the expected number of GRSs as about 2000, for the resolution and sensitivity limits of FIRST, NRAO VLA Sky Survey and Sloan Digital Sky Survey surveys. Compared with earlier compilations, there is a significant increase in the number of large giants with sizes $>2 \mathrm{Mpc}$ as well as those at high redshifts with $\mathrm{z}>1$. We discuss aspects of their evolution and suggest that these are consistent with evolutionary models.
\end{abstract}

Key words: galaxies: active - galaxies: nuclei - galaxies: structure - quasars: general - radio contiuum: galaxies

\section{INTRODUCTION}

The giant radio sources (GRSs) are defined as extragalactic radio sources, hosted by galaxies (giant radio galaxies; GRGs) or quasars (giant radio quasars; GRQs), for which the projected linear size of radio structure is larger than $0.7 \mathrm{Mpc}^{1}$ (assuming $H_{0}=71 \mathrm{~km} / \mathrm{s} / \mathrm{Mpc}, \Omega_{\mathrm{M}}=0.27, \Omega_{\mathrm{vac}}=$ 0.73). The first objects of this type to be found were $3 \mathrm{C} 236$ and DA240, identified by Willis, Strom \& Wilson (1974), while the first sample of 53 GRSs compiled from the literature was published by Ishwara-Chandra \& Saikia (1999). Since that time, the number of these relatively rare sources have been growing in number due to the all-sky surveys and the increasing amount of sensitive and good-quality radio and optical data. Many research groups searched for giants over the entire sky using modern radio surveys

\footnotetext{
* E-mail: cygnus@oa.uj.edu.pl

1 Many earlier authors, assuming $H_{0}=50 \mathrm{~km} / \mathrm{s} / \mathrm{Mpc}$, have used a lower limit of $1 \mathrm{Mpc}$ as the defining size for GRSs. For the currently accepted cosmological parameters as given above, a limiting size of $\sim 0.7 \mathrm{Mpc}$ is appropriate.
}

like the Westerbork Northern Sky Survey (Rengelink et al. 1997 ) at $0.3 \mathrm{GHz}$, the Sydney University Molonglo Sky Survey (SUMSS, Bock, Large \& Sadler 1999) at $0.8 \mathrm{GHz}$, the NRAO VLA Sky Survey (NVSS, Condon et al. 1998) and the Faint Images of the Radio Sky at TwentyCentimeters (FIRST, Becker, White \& Helfand 1995) at 1.4 GHz. The more recent samples by Schoenmakers et al. (2000a), Saripalli et al. (2005), Lara et al. (2001a), Machalski, Jamrozy \& Zoła (2001); Machalski et al. (2006); Machalski, Kozieł-Wierzbowska \& Jamrozy (2007), Kuźmicz \& Jamrozy (2012) as well as Dabhade et al. (2017) and others have increased the number of known GRSs more than 6 times as compared to the sample of Ishwara-Chandra \& Saikia (1999).

However, GRSs may not be easily recognizable if the surface brightness of the lobes is low. This could be the case for the less luminous GRSs with large angular sizes. Also, at high redshifts, where the inverse-Compton losses against the cosmic microwave background radiation are large, extended radio sources could have weak radio lobes (e.g. Konar et al. 2004). In these cases the hot-spots or lobes may not appear to be connected with the radio core, thereby making the de- 
tection of GRSs quite a challenging task. Due to all these reasons as well as the steep spectra of many GRSs, the newgeneration radio interferometers such as the LOw-Frequency ARray (van Haarlem et al. 2013), the Murchison Widefield Array (MWA; Lonsdale et al. 2009), the Square Kilometre Array (SKA; Carilli \& Rawlings 2004) or upgrades of existing low-frequency telescopes such as the Giant Metrewave Radio Telescope (GMRT; Gupta et al. 2017) should be of particular help in finding more of these objects.

From previous studies, it is still unclear why just a small fraction of radio sources reach such large sizes. Nevertheless, owing to the observations conducted in the last decade, our knowledge of the nature of GRSs has progressed significantly. These investigations focused on the role of the properties of the intergalactic medium (IGM; Machalski et al. 2006; Subrahmanyan et al. 2008; Kuligowska et al. 2009), the advanced age of the radio structures (e.g. Mack et al. 1998; Machalski, Jamrozy \& Saikia 2009), recurrent radio activity (e.g. Subrahmanyan, Saripalli \& Hunstead 1996; Schoenmakers et al. 2000a; Saikia \& Jamrozy 2009; Machalski et al. 2011), and specific properties of the central active galactic nuclei (AGNs; e.g. black hole mass and accretion rate, Kuźmicz \& Jamrozy 2012), as underlying reasons for their gigantic sizes. They revealed that GRSs are quite similar to radio sources with smaller radio structures, but significantly older. There is a trend for the spectral ages of radio galaxies to increase with linear size (e.g. Murgia et al. 1999; Parma, Murgia \& Morganti 1999; Murgia 2003; Jamrozy et al. 2008). However, there also exist small-sized radio galaxies with large ages $\left(\sim 10^{8} \mathrm{yr}\right)$. Murgia et al. (2011) presented a study of five very aged radio galaxies with linear sizes of only about $100 \mathrm{kpc}$. Considering the IGM properties, it has been measured that in the vicinity of some GRSs the IGM density is lower (e.g. Schoenmakers et al. 2000b; Lara et al. 2000), while we need to understand why we do observe giants at higher redshifts $(z>1)$ where the IGM density is actually higher than at present. Moreover, Komberg \& Pashchenko (2009) have shown that there is no correlation between the radio source size and the density of galaxies in the neighbourhood. However, we still lack an unequivocal explanation for the giant sizes of some of these radio sources. It is obvious that GRSs are very interesting objects worth intensive research. Investigations of their properties are necessary to fully understand the processes responsible for the formation and evolution of radio sources in general.

Moreover, GRSs can be useful for cosmological studies. They can help in determining the IGM properties at different redshifts. A number of studies carried out during the last several years show that GRSs are a very valuable tool for investigating the large-scale structure of the Universe. Their large sizes provide an opportunity to probe the distribution of the Warm-Hot Intergalactic Medium (WHIM) in filaments of the large-scale structure of the Universe (e.g. Malarecki et al. 2015, 2013; Pirya et al. 2012; Peng, Chen \& Strom 2015). These studies focus on searching interactions of radio lobes with the ambient medium revealed through asymmetries of radio structures and the distribution of neighbouring galaxies. It is believed that through these kinds of investigations it will be possible to find the 'missing' baryons predicted by the Big Bang theory (Peng, Chen \& Strom 2015).
Furthermore, the recent investigations of Bassani et al. (2016) show that a large fraction of soft gamma-ray selected radio sources become GRSs. The all-sky observations of the INTErnational Gamma-Ray Astrophysics Laboratory (INTEGRAL; Winkler 1994) and Swift (Gehrels et al. 2004) satellites reveal a large population of AGNs. The doublelobed radio sources are not too common in this group (about $7 \%$ ), yet it is very intriguing that a large fraction of them are giants $(23 \%)$. It may be the case that high-energy surveys could be more efficient in searching for new GRSs as compared to the radio surveys, where for example, in the wellstudied 3CRR sample about $8 \%$ of the sources are identified as GRSs.

Given the above background, it is clear that it is highly advisable to look for new giants, and compiling a large sample of GRSs will facilitate future research in this field.

In this paper, we present a catalogue of GRSs known to date. We provide their principal parameters as well as characteristics of the sample. In this compilation of GRSs we do not consider objects such as cluster radio relics and/or radio halos that can also exceed our defining size of $700 \mathrm{kpc}$. The content of the paper is as follows. Section 2 describes details of the GRSs catalogue. In Sections 3 and 4 we analyse and discuss their distribution, physical parameters, and aspects of their evolution, while in Section 5 we present our concluding remarks.

\section{CATALOGUE}

For several years we have been browsing the literature, including survey results, and analysing data to search for radio galaxies of large linear sizes. We focused mostly on the existing compilations of giants, as well as published studies of individual sources of such types, but also examined wellstudied samples such as $3 \mathrm{CRR}$ and compilations of structures of radio sources such as, for example, that by Nilsson (1998). In effect we have compiled a list of all GRSs known to the end of 2017. The list and principal parameters of GRSs are presented in Table 1 which is arranged as follows: Col. 1 - source name; Cols. 2 and 3 - J2000.0 coordinates of the GRS host galaxy or quasar; Col. 4 - optical identification ( $\mathrm{G}$ - galaxy or Q - quasar); Col. 5 - redshift; Col. 6 radio morphological type based on the Fanaroff-Riley classification scheme (see below); Col. 7 - angular size in arcmin; Col. 8 - projected linear size, D, in Mpc; Col. $9-r$ band optical aperture magnitude; Col. 10 - total flux-density at 1.4 or 1.388 or 0.843 or $0.325 \mathrm{GHz}$ in units of mJy; Col. 11 - error in total flux-density; Col. $12-1.4 \mathrm{GHz}$ total radio luminosity in W/Hz; Col. 13 - References.

The redshifts enclosed within parentheses correspond to the photometric redshifts taken either from the literature or from the Sloan Digital Sky Survey (SDSS; Albareti et al. 2017). The $1.4 \mathrm{GHz}$ flux-densities were measured mostly on the maps of the NVSS using the AIPS ${ }^{2}$ software and particularly the task 'tvstat'. During the measurements we have made efforts to exclude unrelated (foreground/background) sources superposed on the extent of some GRSs. For some

2 www.aips.nrao.edu/index.shtml 
sources with declination below $-40^{\circ}$, we used the fluxdensity data at $1.388 \mathrm{GHz}$ taken from Saripalli et al. (2012). They are marked as ' $a$ ' in the Table 1 . The SUMSS fluxdensity measurements are marked as ' $b$ ', the measurements at $0.843 \mathrm{GHz}$ given by Saripalli et al. (2005) are marked as letter ' $c$ ' and the measurements at $0.325 \mathrm{GHz}$ given by Sebastian et al. (2018) are marked as letter ' $d$ '. In order to determine the flux-density error, we used the formula given by Klein et al. (2003):

$\delta \mathrm{S}=\sqrt{(\mathrm{S} \cdot 0.03)^{2}+\left(\sigma \cdot \sqrt{\frac{\Omega_{\text {int }}}{\Omega_{\text {beam }}}}\right)^{2}}$,

where ' $\mathrm{S}$ ' is the measured total flux-density in mJy, the 0.03 value corresponds to the assumed $3 \%$ calibration error; ' $\sigma$ ' is the r.m.s. noise measured around the source, ' $\Omega_{\text {int }}$ ' is the integration area, and ' $\Omega_{\text {beam }}$ ' is the beam's solid angle. The radio morphological type (FRI, FRII; Fanaroff \& Riley 1974) was predominantly taken from the literature but for newly classified sources we judged it by ourselves using highresolution radio maps. To determine the total radio luminosity, we used the formula given by Brown, Webster \& Boyle (2001) and assumed for all sources a mean spectral index value as $\alpha=-0.8$ (following e.g. Nilsson 1998). For sources with only 0.843 or $0.325 \mathrm{GHz}$ flux density measurements available we determined the $1.4 \mathrm{GHz}$ flux density using the above given spectral index value.

For classical FRII sources, which constitute about 90 per cent of the sources in our list, we measure the angular extent as the size between the hotspots. Our measurements and those given by other authors are similar for these types of sources. In cases when we do not have an image of good enough angular resolution for a source, we adopt the angular size as was given in the literature. Controversies regarding angular size measurement appear mostly in the case of FRI (and hybrid FRI/FRII) sources. For such objects we try to estimate the angular size from a map/publication at which the source shows a maximal angular radio extent and its size was taken as the distance between the opposite edges. Something similar occures for FRII sources (e.g. J0116-4722, J1548-3216) which show a double-double radio structure (without clear hotspots in the outer lobes) or those that have at least a clear extension beyond the hotspot(s) (e.g. DA240). In the case of sources which have a very curved morphology (e.g. wide-angle-tail, head tail radio galaxies) their angular extent was measured along the source ridge, and were not, as in the case of most giants, taken as the shortest distant between opposite edges.

The optical $r$-band aperture magnitudes are taken from the PanSTARRS data archive (Flewelling et al. 2013) which has surveyed the sky north of $\delta=-30^{\circ}$ in five photometric bands (grizy). For some objects south of $\delta=-30^{\circ}$ we have taken $r$-band magnitudes from Saripalli et al. (2012) and marked them as ' $r$ ' in Table 1.

The references given in Table 1 are mostly related to papers where a radio source has been recognised as a giant for the first time, or to papers where some important parameters characterising a particular object (e.g. redshift, radio map) were published. In Table 1 we include only the confirmed GRSs, though there are many more GRS candidates with redshifts still to be determined.

The final catalogue includes 349 sources, of which 280 are galaxies, 68 are quasars, and 1 is of uncer- tain optical identification, covering the redshift range of $0.016<\mathrm{z}<3.22$. In the sample, there are 46 objects with photometric estimation of redshifts. Redshifts larger than 1 were found for 22 GRSs based on spectroscopic measurements and for 6 GRSs based on photometric estimations. The most distant GRSs are J1145-0033 with redshift $\mathrm{z}=2.055$ (Kuźmicz, Kuligowska \& Jamrozy 2011) and J1235+3925 (i.e. 4C39.37) with redshift $\mathrm{z}=3.22$ (Mack et al. 2005). However, the extent of the radio protrusions of the latter object is not certain, since Mack et al. (2005) mentioned that "it cannot be excluded that these are artefacts caused by an imperfect amplitude calibration". Furthermore, 4C39.37 is the highest luminosity GRS in our sample. Although its structure needs to be confirmed, it is consistent with the extrapolation of the $\log \mathrm{P}-\mathrm{z}$ relation (Figure 6) appearing as the most distant and luminous object.

More than half of the GRSs have projected linear sizes larger than $1 \mathrm{Mpc}$. Four of them have extremely large sizes: J1006+3454 (4.23 Mpc), J0931+3204 (4.29 Mpc), $\mathrm{J} 1234+5318$ (4.44 Mpc), J1420-0545 (4.69 Mpc). The last one is still the largest GRS known to date (Machalski et al. 2008). Most of the catalogued sources have an FRII radio morphology. Only 20 sources reveal an FRI structure and 16 are classified as hybrid FRI/FRII sources.

This sample of GRSs as a whole is quite heterogeneous, including objects from studies in which sources were not selected in any systematic manner. Moreover, it is restricted by the selection effects related to sensitivity of radio and optical surveys. However, it may be possible to construct statistically complete subsamples in restricted areas of the sky that have been imaged uniformly by surveys such as NVSS/FIRST and optical surveys such as SDSS.

\section{SKY COVERAGE}

In Figures 1 and 2 we plot the distributions of GRSs from our sample both in the Galactic and equatorial coordinates. It can be seen that it is not homogeneous and that a large fraction of known giants are seen in the northern hemisphere. On the sky maps there are regions where a lot of giants can be observed and regions without any recognized source (areas marked in gray in Figure 1). In the strip along the Galactic equator $\left(|b| \lesssim 15^{\circ}\right)$, the optical identification of GRSs can be difficult due to higher Galactic extinction. Therefore, infrared surveys (e.g. the Two Micron All-Sky Survey; 2MASS; Skrutskie et al. (2006) or the Wide-field Infrared Survey Explorer (WISE; Wright et al. (2010)) can be helpful for identifying host galaxies in this region. Moreover, there are a number of extended individual radio structures inside the Milky Way Galaxy that may confuse some of the GRSs' radio structure. The regions devoid of GRSs away from the Galactic plane are mostly due to incomplete coverage of both radio and optical surveys. Therefore, it would be good if future optical and radio survey efforts focus primarily on these 'empty' fields for identifications of new GRSs.

The largest number of giants is recognised in the regions that are covered by the FIRST radio survey, along with the availability of optical data (e.g. SDSS). As can be seen in Figure 2, where the surface density of giants in the sky plane is depicted by different colours, the densest region is located in the area defined by $12.8^{\mathrm{h}}<\alpha<14.4^{\mathrm{h}}$ and 
$36^{\circ}<\delta<54^{\circ}$. There are 15 giants in this area, of which 12 are identified with galaxies and the remaining are identified with quasars. Similar numbers of GRSs are expected in other regions observed to similar radio and optical sensitivity limits. Extrapolating this density over the whole sky $(4 \pi \mathrm{sr})$ and assuming a homogeneous distribution of GRSs, the total number of giants should be about 2000. This estimate does not take into account objects with low surface brightness that remain undetectable in the NVSS survey. Also, since most of our sources have been selected from low-frequency surveys (less than about $1.4 \mathrm{GHz}$ ), core-dominated GRSs are also likely to be under-represented in the sample. Therefore, this is just a lower limit to the expected number of GRSs. Furthermore, the regions with the largest numbers of giants coincide with coverage of the FIRST survey, where better radio map resolution allowed for the identification of radio cores associated with host galaxies. However, it should be stressed that apart from regions that are not covered by both radio and optical surveys, there are also relatively large regions where FIRST and SDSS data are available but they still show a low number of GRGs. For example, such regions are placed near the North Galactic pole $\left(12^{\mathrm{h}}<\alpha<14.4^{\mathrm{h}}\right.$, $0^{\circ}<\delta<18^{\circ}$ with density of only 2 GRSs over $317.33 \mathrm{deg}^{2}$ ) and in an area bounded by $21.8^{\mathrm{h}}<\alpha<3.6^{\mathrm{h}}, 0^{\mathrm{o}}<\delta<18^{\mathrm{o}}$ (with an average density of 2.6 GRSs over $317.33 \mathrm{deg}^{2}$ ). The deficiency of known GRSs in those particular regions is not because of a lack of radio and optical data but due to a lack of conducting any systematic surveys for giants.

\section{DISCUSSION}

\subsection{Physical parameters of the GRSs}

In Figure 3, we present distributions of redshifts, projected linear sizes and $1.4 \mathrm{GHz}$ total radio luminosities for the GRSs catalogued in this paper. The linear sizes range from the cut-off value of $700 \mathrm{kpc}$ to $4.69 \mathrm{Mpc}$, with a median value of $1.14 \mathrm{Mpc}$. In the earlier compilation by Ishwara-Chandra \& Saikia (1999) the largest source excluding $3 \mathrm{C} 236$, was $8 \mathrm{C} 0821+695$ which in our present cosmology has a projected linear size of $2.54 \mathrm{Mpc}$. In the present catalogue there are 13 sources with a size of at least $2.5 \mathrm{Mpc}$, a substantial increase that enable us to investigate the evolutionary status of these large sources. As seen in Figure 3 (middle panel), the FRII GRSs are mostly larger than the FRI GRSs. The median values of projected linear size of FRII, FRII/FRI and FRI type GRSs are 1.15, 1.18 and 0.99 $\mathrm{Mpc}$, respectively. This indicates that the FRII radio sources tend to be larger than FRIs. Similar results were obtained by, e.g. Wing \& Blanton (2011), who studied distributions of the projected linear size of the radio sources in clusters of galaxies.

There has also been a substantial increase in the number of high-redshift objects, say those with $\mathrm{z}>1$. The median redshift is 0.24 with the highest value being 3.22 . There are 28 objects with a redshift of at least 1 , while there were none in the earlier compilation by Ishwara-Chandra \& Saikia (1999). Although a large number of larger sources at high redshifts have been discovered from the new, more sensitive surveys, most giants are relatively nearby objects with sizes close to $1 \mathrm{Mpc}$ and $1.4 \mathrm{GHz}$ total luminosity $\log \mathrm{P}_{\text {tot }}[\mathrm{W}$
$\mathrm{Hz}^{-1}$ ] ranging from $\sim 23.0$ to 28.3 . The mean value of $\log \mathrm{P}_{\text {tot }}\left[\mathrm{W} \mathrm{Hz}^{-1}\right]=25.5$ for giants is slightly higher than the mean $1.4 \mathrm{GHz}$ total radio luminosity of smaller sized FRII's (from the sample of Koziel-Wierzbowska \& Stasińska 2011), which is equal to $\log \mathrm{P}_{\text {tot }}\left[\mathrm{W} \mathrm{Hz}^{-1}\right]=25.1$.

\subsection{Hubble diagram for the GRSs}

Although almost all the redshifts are spectroscopic, a few listed within brackets are photometric. Figure 4 shows the $r$-band apparent magnitude as a function of spectroscopic redshift, for the catalogued objects. This relation is known as the "Hubble diagram" and can be used to estimate the "photometric" redshift of galaxies lacking spectroscopic observations. Subsequently, we performed a linear regression fit to the PanSTARRS data points (excluding all objects with quasar hosts from this analysis), and obtained the following relation:

$r_{\text {mag }}=(3.91 \pm 0.18) \log (z)+(21.11 \pm 0.15)$

with a correlation coefficient of 0.89 . Such a relation is consistent with the results obtained by Eales (1985) for a set of $3 \mathrm{CRR}$ galaxies, and suggests that the estimated redshifts are reasonably reliable. A similar relation but with a steeper slope, $r_{\text {mag }}=(8.83 \pm 0.35) \log (\mathrm{z})+(22.96 \pm 0.37)$ was obtained for 81 large angular sized radio galaxies by Lara et al. (2001b). The differences between our and Lara et al. (2001b) estimates are probably because of the different filter characteristics used during observations. For the low-redshift objects (mostly the brightest galaxies) the magnitudes from Lara et al. (2001b) are up to 3 mag brighter than those from PanSTARRS.

\subsection{The luminosity-linear size $(\mathrm{P}-\mathrm{D})$ diagram}

The radio luminosity-linear size ( $\mathrm{P}-\mathrm{D})$ diagram has been used by many researchers in the past (e.g. Kaiser, Dennett-Thorpe \& Alexander 1997; Ishwara-Chandra \& Saikia 1999; Blundell, Rawlings \& Willott 1999; Machalski, Chyży \& Jamrozy 2004) as a useful tool to study the evolution of radio sources. In Figure 5 we plot the $\mathrm{P}-\mathrm{D}$ relation for the sample of GRSs and smallersized $3 \mathrm{CRR}^{3}$ (Laing, Riley \& Longair 1983) and FRII radio sources taken from Koziel-Wierzbowska \& Stasińska (2011). We also superimpose the evolutionary tracks proposed by Kaiser, Dennett-Thorpe \& Alexander (1997) for three different jet powers. For the 3CRR sample the flux densities measured at $0.178 \mathrm{GHz}$ were extrapolated to the frequency of $1.4 \mathrm{GHz}$ using the spectral index of individual sources and then the total radio luminosities were calculated using the formula given by Brown, Webster \& Boyle (2001). Thirteen of the radio sources from the $3 \mathrm{CRR}$ sample, and 21 radio sources from the FRII sample have sizes larger than $0.7 \mathrm{Mpc}$; these are included in the sample of GRSs.

The models (e.g. Kaiser, Dennett-Thorpe \& Alexander 1997; Ishwara-Chandra \& Saikia 1999; Blundell, Rawlings \& Willott 1999; Machalski, Chyży \& Jamrozy 2004) all suggest that

3 http://www.jb.man.ac.uk/atlas 


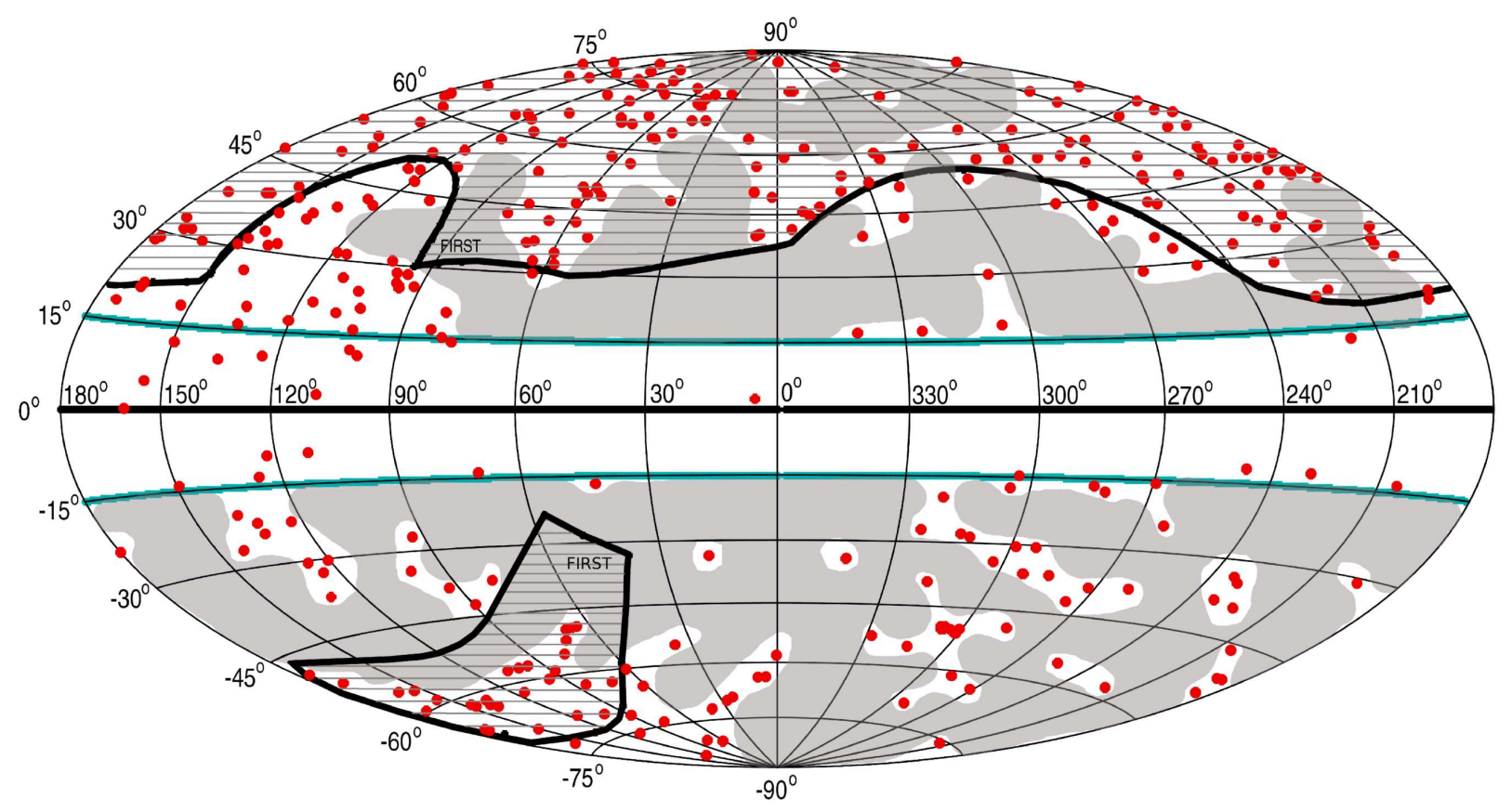

Figure 1. Distribution of GRSs in the plane of the sky in Galactic coordinates. The blue lines are plotted at Galactic latitudes $b= \pm 15^{\circ}$ denoting the larger Galactic extinction regions. In gray we coloured the regions outside the Galactic plane where no GRSs have been found. The sky area that is covered by the FIRST survey is framed by a thick black curve and in addition the area is hatched.

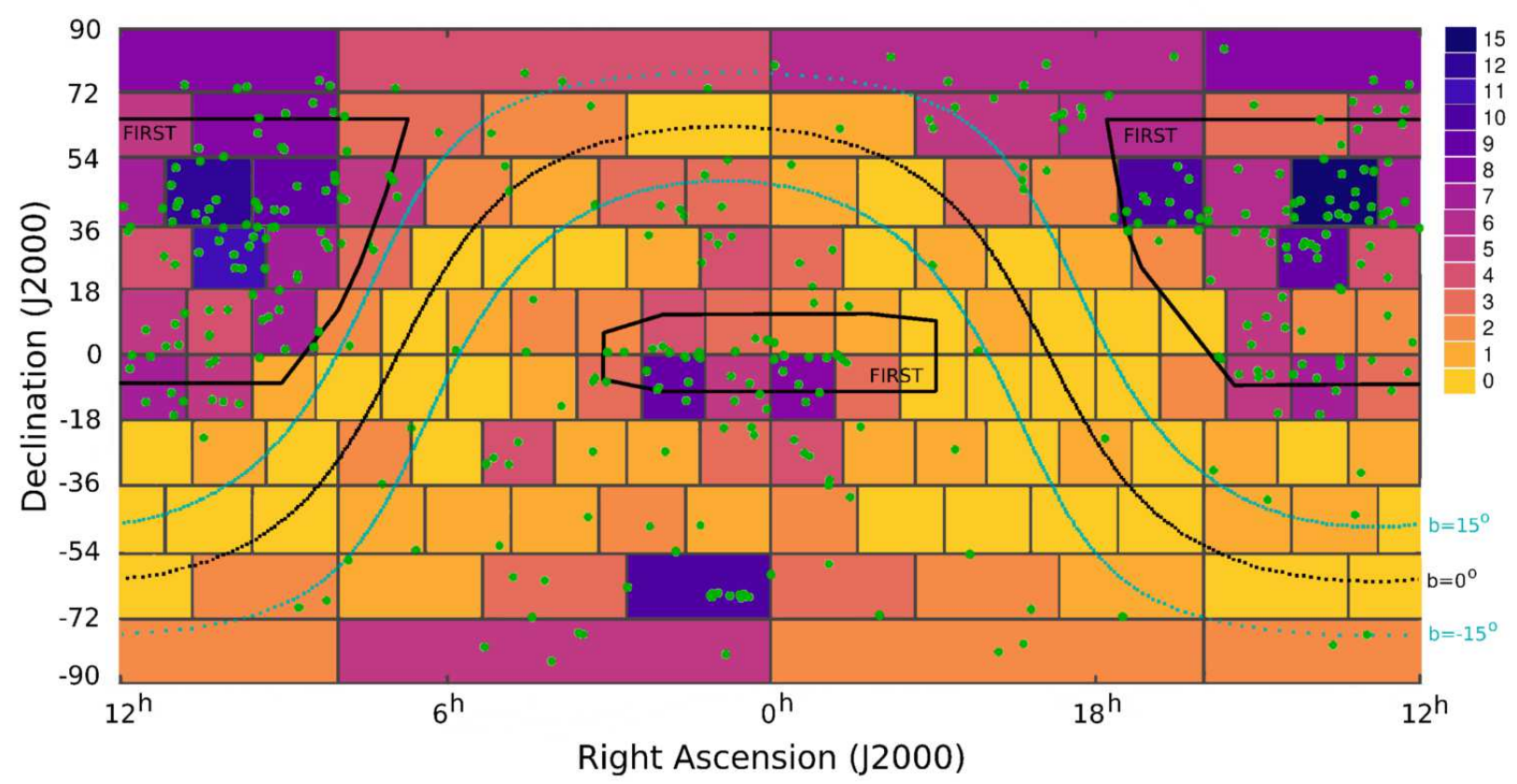

Figure 2. The distribution of GRSs in the plane of the sky in equatorial coordinates. The blue dotted lines correspond to the Galactic latitudes $\mathrm{b}= \pm 15^{\circ}$ and the black dotted line corresponds to the Galactic equator. The different colours denote the number of giants counted inside a particular rectangle and every rectangle covers a the same area of $317.33 \mathrm{deg}^{2}$ (Malkin 2016).

the GRSs are the evolved counterparts of smaller, younger, and more luminous radio sources. The $\mathrm{P}-\mathrm{D}$ diagram shows more clearly a declining upper envelope than was noted by Ishwara-Chandra \& Saikia (1999). The lack of radio sources that have both large sizes and high radio luminosities in our plot is consistent with model expectations of the evolution of radio sources. It has been postulated that GRSs evolve from normal FRII and FRI radio sources (e.g. Ishwara-Chandra \& Saikia 1999), with sources of different jet powers following different evolutionary tracks in the $\mathrm{P}-\mathrm{D}$ 

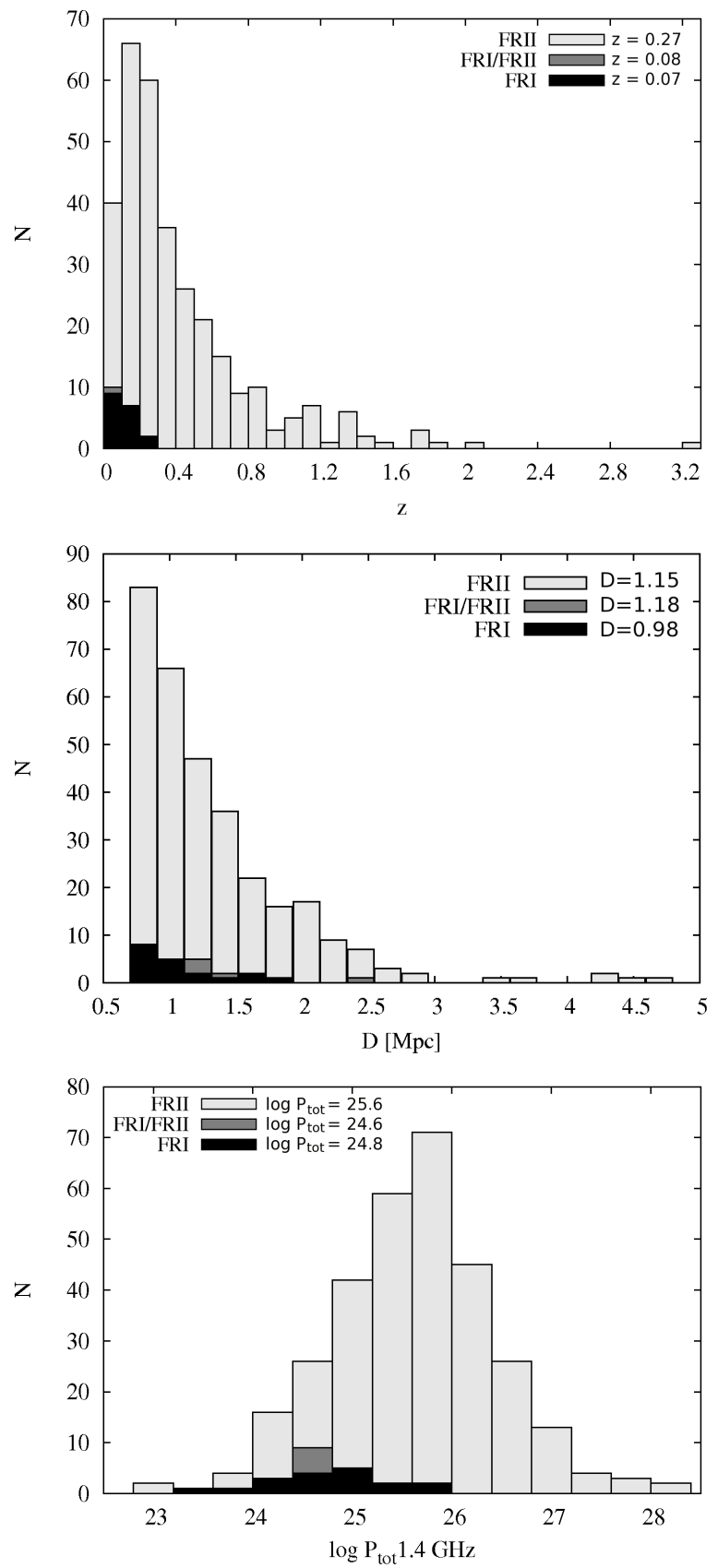

Figure 3. Distributions of redshift (top panel), projected linear size (middle panel), and $1.4 \mathrm{GHz}$ total radio luminosity (bottom panel) for GRSs. The number of sources $\mathrm{N}$ is put on the vertical axis. The values of redshift, projected linear size, and total radio luminosity given on the panels denote the median values for each morphological class of radio sources.

diagram. The upper envelope corresponds to a jet power of $\sim 10^{40} \mathrm{~W}$. With the discovery of many high-redshift, luminous giant sources, the median value of total radio luminosities of the GRSs, $\log P_{t o t}\left[\mathrm{~W} \mathrm{~Hz}{ }^{-1}\right] \sim 25.5$ is somewhat higher than that of typical FRII radio sources (from the sample of Koziel-Wierzbowska \& Stasińska 2011) for which we obtained a median of $\log P_{t o t}[\mathrm{~W}$ $\left.\mathrm{Hz}^{-1}\right]=25.1$. This fact, and evolutionary tracks from

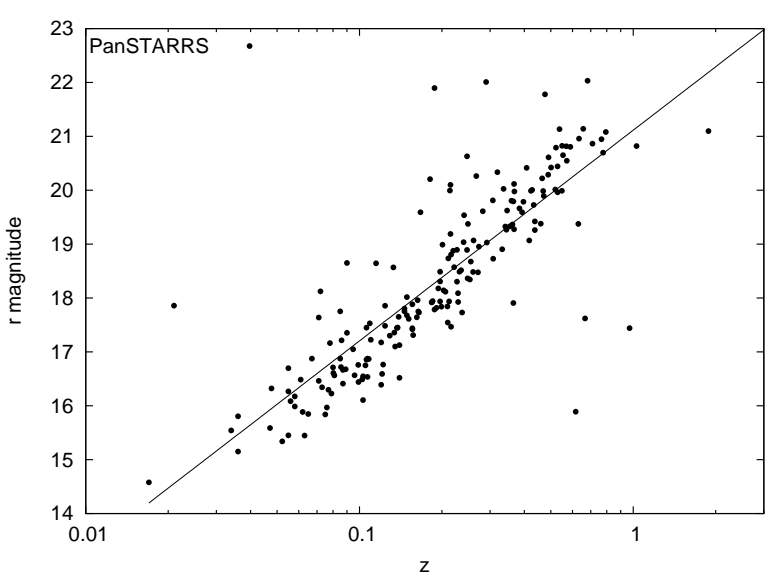

Figure 4. Correlation between $r$-band apparent PanSTARRS magnitude and redshift. The solid line corresponds to the best fit obtained for data points.

Kaiser, Dennett-Thorpe \& Alexander (1997), indicate that the high-luminosity GRSs evolve possibly from the most luminous radio sources like those from the 3CRR sample for which the median of $\log P_{t o t}\left[\mathrm{~W} \mathrm{~Hz}^{-1}\right]$ is 26.4. Less-luminous GRSs can evolve from lower-luminosity FRII and FRI radio sources. However, we observe the deficit of low-luminosity and high-redshift GRSs visible in Figure 6 where we plot the total radio luminosity as a function of redshift, as would be expected in case of a Malmquist bias. However, in the $\mathrm{P}-\mathrm{D}$ diagram (Figure 5) just as there appears to be a rough upper envelope for the GRSs, the Figure 5 also suggests a rough lower envelope. The largest GRSs are not of the lowest luminosities amongst the GRSs. This apparent deficit of large, low-luminosity GRGs, is possibly due to a combination of selection effects (e.g. low surface-brightness of extended sources) and quenching due to inverse-Compton scattering.

\subsection{High-redshift GRSs}

As noted earlier, there are 28 very distant GRSs with redshift $z>1$ in our sample. However, for a long time GRSs were not expected to be found at high redshift. According to Kapahi (1989) since the density of the IGM increases as $\rho_{I G M} \propto(1+z)^{3}$, the radio lobe expansion could be significantly hampered at high redshifts. This explains the smaller median size (equal to $0.899 \mathrm{Mpc}$ ) of high-redshift giants in comparison with $\mathrm{z}<1$ GRSs for which the median size is $1.16 \mathrm{Mpc}$. In addition, the surface brightness decreases with redshift as $(1+z)^{-4}$. This makes it difficult to recognise extended radio lobes and thus GRSs themselves in earlier cosmological epochs. Nevertheless, the number of high-redshift GRSs has grown due to sensitive radio surveys. We examine further the dependence of median size on redshift by considering equal number of GRSs in each redshift bin, and also considering redshift bins of equal width (Figure 7). While in both cases there is a decrease in the median size with redshift for $\mathrm{z}>0.5$, the median size increases with redshift in the range $0<\mathrm{z}<0.5$. Earlier studies of the linear size evolu- 


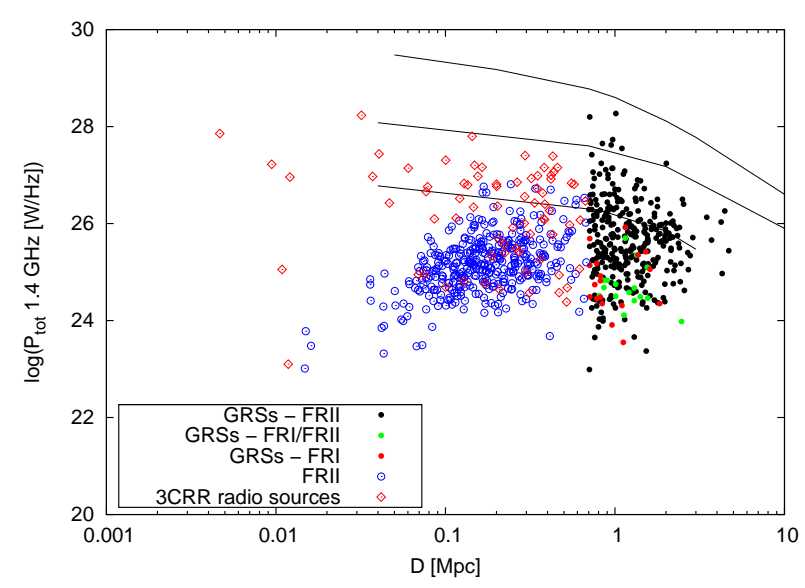

Figure 5. P-D diagram for GRSs (dots), 3CRR radio sources (diamonds) from Laing, Riley \& Longair (1983), and FRII radio sources from Koziel-Wierzbowska \& Stasińska (2011) (pluses). The solid lines (from top to bottom) represent the evolutionary scenarios from Kaiser, Dennett-Thorpe \& Alexander (1997) for jet powers of $1.3 \times 10^{40} \mathrm{~W}$ at $\mathrm{z}=2,1.3 \times 10^{39} \mathrm{~W}$ at $\mathrm{z}=0.5$ and $1.3 \times 10^{38} \mathrm{~W}$ at $\mathrm{z}=0.2$.

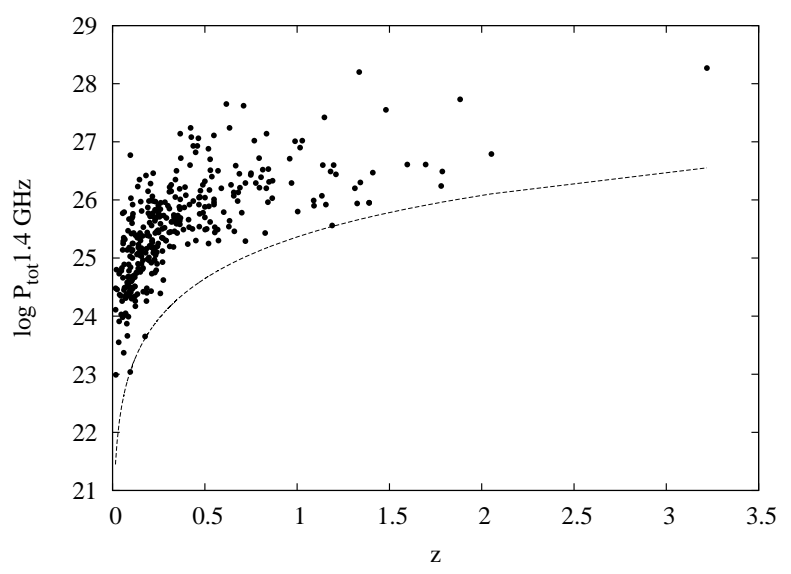

Figure 6. The total radio luminosity at $1.4 \mathrm{GHz}$ as a function of redshift for a sample of GRSs. The dashed line represents the total radio luminosity of the radio source with flux density of $\mathrm{S}_{1.4 \mathrm{GH} z}$ $=5 \mathrm{mJy}$, corresponding to the lowest flux density of sources in our catalogue.

tion of extragalactic radio sources have reported a decrease in median linear size with redshift for both bright and faint source samples (Neeser et al. 1995; Singal 1996) of the form $\mathrm{D} \propto(1+z)^{-n}$, where $\mathrm{n}$ was found to have a range of values from about 1 to 3 . These were consistent with the expectations of theoretical models (Kaiser \& Alexander (1999). However, such studies are complicated by possible correlations of size with luminosity, which may be different for galaxies and quasars (e.g. Singal 1988). Authors (e.g. Singal 1996) have stressed the importance of examining the $D_{-}-z$ relationship for sources of similar radio luminosity, and perhaps separately for different classes. This would be possible for a large uniformly selected sample of giant sources, which is beyond the scope of the present paper. However, it may be relevant to note the recent paper by Onah et al. (2018) for a sample consisting of both galaxies and quasars, where

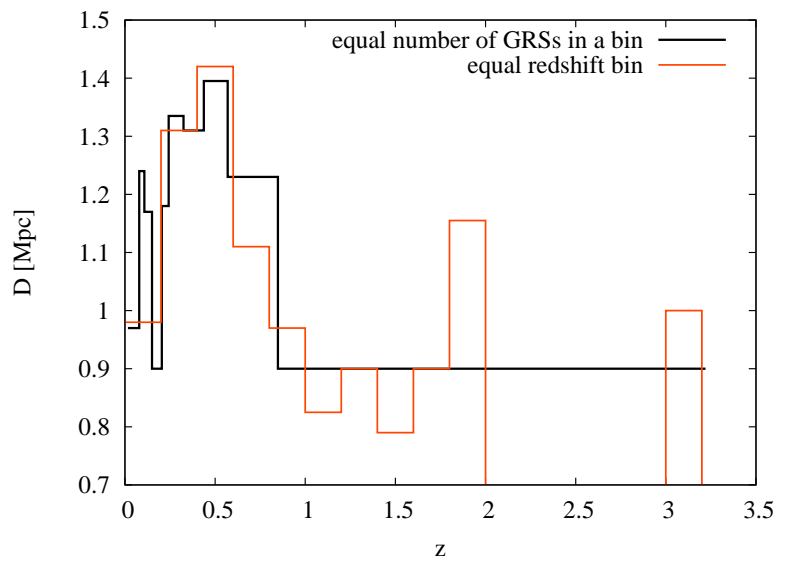

Figure 7. The GRS's median size distribution as a function of redshift. Black line: the bins contain the same number of GRSs 35 sources each (except the last redshift bin which has 34 sources); red line: the bins are of equal redshift - bin size is equal to 0.2 .

they report an increase in median size with redshift up to about $\mathrm{z}=1$. In the context of GRSs one also needs to examine whether large diffuse low-luminosity giants may be below the thresholds of existing surveys and observations.

The largest sample of GRSs within the redshift range $1<\mathrm{z}<2$ was presented by Kuligowska et al. (2009). Considering the dynamical evolution of radio galaxies, these authors concluded that the giant sizes of most distant objects are related rather to the very high power (up to $10^{40} \mathrm{~W}$ ) of their jets, which overcomes the higher density of the IGM. These values are consistent with the location of these sources in the $\mathrm{P}-\mathrm{D}$ diagram and the evolutionary models of radio sources.

\subsection{GRSs in regions of cosmic voids}

Furthermore, to examine the dependence of GRSs occurrence on the IGM density, we correlated the GRS locations with the positions of 1228 cosmic voids provided by Mao, Berlind \& Scherrer (2017). The catalogue of voids was compiled using the Baryon Oscillation Spectroscopic Survey (Dawson et al. 2013), which is a part of the SDSS-III (Eisenstein et al. 2011). The survey covers the sky area in a declination range of $-8^{\circ}<\delta<66^{\circ}$ and contains voids with Galactic latitude $|\mathrm{b}|>20^{\circ}$ and in a redshift range of $0.2<\mathrm{z}<0.67$. Within the sky area and redshift range covered by this void catalog, there are 91 GRGs of our compilation. However, we found just one clear correlation: the GRG J1355+2923 is located only about $17 \mathrm{Mpc}$ from the centre (RA: $13^{h} 51^{m} 58^{\mathrm{s}} .08$ Dec: $29^{\circ} 05^{\prime} 34^{\prime \prime} .8$, J2000.0) of a void named 'CMASS North 19434' at $\mathrm{z}=0.501$. The effective radius of the void is $40.5 \mathrm{Mpc}$. Although there have been a number of suggestions that GRSs occur in regions of low IGM density (e.g. Machalski et al. 2006; Kuligowska et al. 2009, Chen et al. 2011, Malarecki et al. 2015), the above result suggests that the size of GRGs is not related solely to the galaxy density around their host galaxies. Apart from deep X-ray observations, deeper optical observations including improved photometric redshifts (e.g. from PanSTARRS) could provide better estimates of the ambient galaxy density of GRGs using the methods ap- 
plied by Komberg \& Pashchenko (2009), but out to higher redshifts.

\section{CONCLUDING REMARKS}

We present the largest-to-date sample of GRSs (defined here as having a projected largest linear size of at least $0.7 \mathrm{Mpc}$ ), which consists of 349 individual objects. It is limited by sensitivity and coverage of the available radio and optical surveys in different parts of the sky. Analysing the distribution of GRSs in the celestial plane, we distinguish sky regions that are underdense in GRSs and that should be explored for further objects yet to be identified even with current sensitivity limits. We estimate the lower limit to the number of GRSs on current sensitivity limits as 2000, based on the density of known GRSs in the regions where most such sources have been observed.

The present sample has considerably increased the number of GRSs with projected sizes larger than about $2 \mathrm{Mpc}$, and also at high redshifts of greater than $\sim 1$. The Hubble diagram for the galaxies in the GRS sample has been found to be similar to that of 3CRR radio galaxies, with the $r$-band magnitude being well correlated with redshift, allowing for quick estimates of photometric redshifts for giants that have not been spectroscopically observed.

We have examined the luminosity-linear size diagram with the enlarged sample, and find the upper envelope to be well-defined for the GRSs. This is consistent with the evolution of the radio sources with the highest jet powers of $\sim 10^{40} \mathrm{~W}$. The distribution is the result of different sources with different jet powers, as suggested by evolutionary scenarios (e.g. Kaiser, Dennett-Thorpe \& Alexander 1997; Blundell, Rawlings \& Willott 1999; Ishwara-Chandra \& Saikia 1999; Machalski, Chyży \& Jamrozy 2004).

The high-redshift giants that are also amongst the more luminous ones can be understood in terms of their high jet powers $\left(\sim 10^{40} \mathrm{~W}\right)$, which overcomes the effects of higher ram pressure due to increased density of the IGM. This is consistent with the results of the $\mathrm{P}-\mathrm{D}$ diagram.

While GRSs have been suggested to occur often in regions of low galaxy density, correlating our catalogue of GRSs with cosmic voids, we find only one clear association. Future X-ray telescopes will be useful for probing their environments from sensitive observations.

\section{ACKNOWLEDGMENTS}

We thank the reviewer Prof. H. Andernach for his very detailed and valuable comments that helped improve the paper significantly. A part of this project was supported by the Polish National Center of Science under decision UMO2016/20/S/ST9/00142.

\section{REFERENCES}

Albareti, F. D., Allende, P. C., Almeida, A., et al., 2017, ApJS, 233,25

Amirkhanyan, V. R., Afanasiev, V. L., \& Moiseev, A. V., 2015, AstBu, 70, 45
Andernach, H., Feretti, L., Giovannini, G., et al., 1992, A\&AS, 93,331

Andernach, H., Jiménez Andrade, E. F., Maldonado Sánchez, R. F., \& Vásquez Báez, I. R., 2012, Proceedings of the Science from the Next Generation Imaging and Spectroscopic Surveys Conference, 15-18 October 2012. Online at http://www.eso.org/sci/meetings/2012/surveys2012/posters.html, id. P1

Bankowicz, M., Koziel-Wierzbowska, D., \& Machalski, J., 2015, Proceedings of the SALT Science Conference (SSC2015), 1-5 June 2015. Stellenbosch Institute of Advanced Study. South Africa, Online at http://pos.sissa.it/cgibin/reader/conf.cgi? confid $=250$, id. 34

Bassani, L., Venturi, T., Molina, M., et al., 2016, MNRAS, 461, 3165

Baum, S., A., \& Heckman, T., 1989, ApJ, 336, 681

Becker, R. H., White, R. L., \& Helfand,D. J., 1995, ApJ, 450, 559

Best, P. N., Kauffmann, G., Heckman, T. M., \& Ivezić, Z., 2005, MNRAS, 362, 9

Bhatnagar, S., Krishna, G., \& Wisotzki, L., 1998, MNRAS, 299, 25

Blundell, K. M., Rawlings, S., \& Willott, C. J., 1999, AJ, 117, 677

Bock, D. C.-J., Large, M. I., \& Sadler, E. M., 1999, AJ, 117, 1578

Brescia, M., Cavuoti, S., \& Longo, G., 2015, MNRAS, 450, 3893

Brown, M. J. I., Webster, R. L., \& Boyle, B. J., 2001, AJ, 121, 2381

Carilli, C. L., \& Rawlings, S., 2004, NewAR, 48, 979

Chen, R., Peng, B., Strom, R. G., \& Wei, J., 2011, MNRAS, 412, 2433

Clarke, A. O., Heald, G., Jarrett, T., et al., 2017, A\&A, 601, 25

Colafrancesco, S., Mhlahlo, N., Jarrett, T., Oozeer, N., \& Marchegiani, P., 2016, MNRAS, 456, 512

Condon, J. J., Cotton, W. D., Greisen, E. W., \& Yin, Q. F., 1998, AJ, 115, 1693

Cotter, G., Rawlings, S., \& Saunders, R., 1996, MNRAS, 281, 1081

Coziol, R., Andernach, H., Torres-Papaqui, J. P., OrtegaMinakata, R. A., \& Moreno del Rio, F., 2017, MNRAS, 466, 921

Dabhade, P., Gaikwad, M., Bagchi, J., et al., 2017, MNRAS, 469, 2886

Dawson, K. S., Schlegel, D. J., Ahn, C. P., et al., 2013, AJ, 145, 10

de Bruyn, A. G., 1989, A\&A, 226, 13

Djorgovski, S., Thompson, D. J, Vigotti, M., \& Grueff, G., 1990, PASP, 102, 113

Djorgovski, S. G., Thompson, D. J, Maxfield, L., Vigotti, M., \& Grueff, G., 1995, ApJS, 101, 255

Eales, S. A., 1985, MNRAS, 213, 899

Eisenstein, D. J., Weinberg, D. H., Agol, E., et al., 2011, AJ, 142, 72

Falco, E. E., Kurtz, M. J., Geller, M. J., et al., 1999, PASP, 111, 438

Fanaroff, B. L., \& Riley, J. M., 1974, MNRAS, 167, 31

Filipovic, M. D., Cajko, K. O., Collier, J. D., \& Tothill, N. F. H., 2013, SerAJ, 187, 1

Flewelling, H. A., Magnier, E. A., Chambers, K. C., et al., 2016, 2016arXiv161205243F

Fomalont, E. B., \& Bridle, A. H., 1978, AJ, 83, 725

Gehrels, N., Chincarini, G., Giommi, P., et al., 2004, ApJ, 611, 1005

Gregg, M. D., Becker, R. H., \& de Vries, W., 2006, ApJ, 641, 210

Gupta, Y., Ajithkumar, B., Kale, H. S., et al., 2017, Current Science, 113, 707

Hagen, H.-J., Engels, D., \& Reimers, D., 1999, A\&AS, 134, 483

Hes, R., de Vries, W. H., \& Barthel, P. D, 1995, A\&A, 299, 17

Hintzen, P., Ulvestad, J., \& Owen, F., 1983, AJ, 88, 709 
Hota, A., Sirothia, S. K., Ohyama, Y., et al., 2011, MNRAS, 417L, 36

Högbom, J. A., 1979, A\&AS, 36, 173

Hunik, D., \& Jamrozy, M., 2016, ApJ, 817, 1

Hurley-Walker, N., Johnston-Hollitt, M., Ekers, R., et al., 2015, MNRAS, 447, 2468

Ishwara-Chandra, C. H., \& Saikia, D. J., 1999, MNRAS, 309, 100

Jägers, W.J., 1986, PhD thesis, University of Leiden

Jamrozy, M., Konar, C., Machalski, J., \& Saikia, D. J., 2008, MNRAS, 385, 1286

Jones, P. A., \& McAdam, W. B., 1992, ApJS, 80, 137

Jones, D. H., Read, M. A., Saunders, W., et al., 2009, MNRAS, 399,683

Kapahi, V.K. 1989, AJ, 97, 1

Kapahi, V. K., Athreya, R. M., van Breugel, W., McCarthy, P.J., \& Subrahmanya, C.R., 1998, ApJS, 118, 275

Kaiser, C. R., \& Alexander, P., 1999, MNRAS, 302, 515

Kaiser, C. R., Dennett-Thorpe, J., \& Alexander, P., 1997, MNRAS, 292, 723

Kharb, P., O'Dea, C. P., \& Baum, S. A., 2008, ApJS, 174, 74

Klein, U., Mack, K.-H., Gregorini, L., \& Vigotti, M., 2003, A\&A, 406, 579

Konar, C., Saikia, D. J., Ishwara-Chandra, C. H., \& Kulkarni, V. K., 2004, MNRAS, 355, 845

Konar, C., Saikia, D. J., Jamrozy, M., \& Machalski, J., 2006, MNRAS, 372, 693

Kozieł-Wierzbowska, D., \& Stasińska, G., 2011, MNRAS, 415, 1013

Kronberg, P. P., Wielebinski, R., \& Graham, D. A., 1986, A\&A, 169,63

Komberg, B. V., \& Pashchenko, I. N., 2009, ARep, 53, 1086

Kuligowska, E., Jamrozy, M., Kozieł-Wierzbowska, D., \& Machalski, J., 2009, AcA, 59, 431

Kuźmicz, A., Kuligowska, E., \& Jamrozy, M., 2011, AcA, 61, 71

Kuźmicz, A., \& Jamrozy, M., 2012, MNRAS, 426, 851

Lacy, M., Rawlings, S., \& Warner, P. J., 1992, MNRAS, 256, 404

Lacy, M., Rawlings, S., Saunders, R., \& Warner P. J., 1993, MNRAS, 264, 721

Lacy, M., Rawlings, S., Hill, G. J., et al., 1999, MNRAS, 308, 1096

Lacy, M., 2000, ApJ, 536, 1

Laing, R. A., Riley, J. M., \& Longair, M. S., 1983, MNRAS, 204, 151

Lara, L., Márquez, I., Cotton, W. D., et al., 1999, A\&A, 348, 699

Lara, L., Mack, K. H., Lacy, M., et al., 2000, A\&A, 356, 63

Lara, L., Cotton, W. D., Feretti, L., et al., 2001a, A\&A, 370, 409

Lara, L., Marquez, I., Cotton, W. D., et al., 2001b, A\&A, 378, 826

Law-Green, J. D. B., Eales, S. A., Leahy, J. P., Rawlings, S., \& Lacy, M., 1995, MNRAS, 277, 995

Leahy, J. P., \& Perley, R. A., 1991, AJ, 102, 537

Lonsdale, C. J., Cappallo, R. J., Morales, M. F., et al., 2009, IEEEP, 97, 1497

Machalski, J., 1998a, A\&AS, 128, 153

Machalski, J., \& Condon, J. J., 1999, APJS, 123, 41

Machalski, J., Chyży, K. T., \& Jamrozy, M., 2004, AcA, 54, 391

Machalski, J., Jamrozy, M., \& Saikia, D. J., 2009, MNRAS, 395, 812

Machalski, J., Jamrozy, M., \& Zoła, S., 2001, A\&A, 371, 445

Machalski, J., Jamrozy, M., Zoła, S., \& Kozieł, D., 2006, A\&A, 454,85

Machalski, J., Kozieł-Wierzbowska, D., \& Jamrozy, M., 2007, AcA, 57, 227

Machalski, J., Kozieł-Wierzbowska, D., Jamrozy, M., \& Saikia, D. J., 2008, ApJ, 679, 149

Machalski, J., Jamrozy, M., Stawarz, Ł., \& Kozieł-Wierzbowska, D., 2011, ApJ, 740, 58
Mack, K. H., Klein, U., O’Dea, C. P., \& Willis, A. G., 1997, A\&AS, 123, 423

Mack, K.-H., Klein, U., O’Dea ,C. P., Willis, A. G., \& Saripalli, L., 1998, A\&A, 329, 431

Mack, K. H., Vigotti, M., Gregorini, L., et al., 2005, A\&A, 435, 863

Malarecki, J. M., Jones, D. H., Saripalli, L., Staveley-Smith, L., \& Subrahmanyan, R., 2015, MNRAS, 449, 955

Malarecki, J. M., Staveley-Smith, L., Saripalli, L., et al., 2013, MNRAS, 432, 200

Malkin, Z., 2016, "A new method to subdivide a spherical surface into equal-area cells", arXiv:1612.03467

Mao, Q., Berlind, A. A., \& Scherrer, R. J., 2017, ApJ, 835, 161

Marecki, A., Jamrozy, M., \& Machalski, J., 2016, MNRAS, 463, 338

Masetti, N., Parisi, P., \& Palazzi, E., 2013, A\&A, 556, 120

McCarthy, P. J., Miley, G. K., de Koff, S., et al., 1997, ApJS, 112, 415

Molina, M., Bassani, L., Malizia, A., et al., 2014, A\&A, 565, 2

Molina, M., Venturi, T., Malizia, A., et al., 2015, MNRAS, 451, 2370

Murgia, M., Fanti, C., Fanti, R., et al., 1999, A\&A, 345, 769

Murgia, M., 2003, Publ. Astron. Soc. Aust., 20, 19

Murgia ,M., Parma, P., Mack, K.-H., et al., 2011, A\&A, 526, 148

Neeser, M. J., Eales, S. A., Law-Green, J. D., Leahy, J. P., \& Rawlings, S., 1995, ApJ, 451, 76

Nilsson, K., 1998, A\&AS, 132, 31

Onah, C. I., Ubachukwu, A. A., Odo, F. C., \& Onuchukwu, C. C., 2018, RMxAA, 54, 271

Owen, F. N, Ledlow, M. J., Eilek, J. A., et al., 2002, IAUS, 199, 171

Parma, P., de Ruiter, H. R., Mack, K. H., et al., 1996, A\&A, 311, 49

Parma, P., Murgia, M., \& Morganti, R., 1999, A\&A, 344, 7

Peng, B., Chen, R.-R., \& Strom, R., 2015, "Giant radio galaxies as probes of the ambient WHIM in the era of the SKA" in proc. Advancing Astrophysics with the Square Kilometre Array, PoS(AASKA14)109, arXiv:1501.00407

Pirya, A., Saikia, D. J., Singh, M., \& Chandola, H. C., 2012, MNRAS, 426, 758

Proctor, D. D., 2011, ApJS, 194, 31

Proctor, D. D., 2016, ApJS, 224, 18

Reid, R. I., Kronberg, P. P., \& Perley, R. A., 1999, ApJS, 124, 285

Rengelink, R. B., Tang, Y., de Bruyn, A. G., et al., 1997, A\&AS, 124,259

Rentería Macario, J., \& Andernach, H., 2017, arXiv171010731R

Riley, J. M. A., Warner, P. J., Rawlings, S., et al., 1989, MNRAS, 236,13

Röttgering, H. J. A., Tang, Y., Bremer, M. A. R., et al., 1996, MNRAS, 282, 1033

Sadler, E. M., Jackson, C. A., Cannon, R. D., et al., 2002, MNRAS, 329, 227

Sadler, E. M., Cannon, R. D, \& Mauch, T., 2007, MNRAS, 381, 211

Saikia, D. J., \& Jamrozy, M., 2009, BASI, 37, 63

Saikia, D. J., Konar, C., \& Kulkarni, V. K., 2006, MNRAS, 366, 139

Santiago-Bautista, I., Rodriguez-Rico, C. A., Andernach, H., et al., 2016, in The Universe of Digital Sky Surveys, Vol. 42, ed. Napolitano N.R., Longo G., Marconi M., Paolillo M., Iodice E., ASSP, 42, 231

Saripalli, L., Gopal-Krishna, R. W., \& Kuehr, H., 1986, A\&A, 170,20

Saripalli, L., Subrahmanyan, R., \& Hunstead, R. W., 1994, MNRAS, 269, 37

Saripalli, L., Patnaik, A. R., Porcas, R. W., \& Graham, D. A., 1997, A\&A, 328, 78 
Saripalli, L., Hunstead, R. W., Subrahmanyan, R., \& Boyce, E., 2005, AJ, 130, 896

Saripalli, L., Subrahmanyan, R., Laskar, T., \& Koekemoer, A., 2008, in From Planets to Dark Energy: The Modern Radio Universe, Proceedings of Science, 130 (astro-ph: 0806.3518)

Saripalli, L., Subrahmanyan, R., Thorat, K., et al., 2012, ApJS, 199, 27

Saunders, R., Baldwin, J. E., \& Warmer, P. J., 1987, MNRAS, 225,713

Schoenmakers, A. P, Mack, K. H., Lara, L., et al., 1998, A\&A, 336,455

Schoenmakers, A. P., de Bruyn, A. G., Röttgering, H. J. A., van der Laan, H., \& Kaiser, C. R., 2000a, MNRAS, 315, 371

Schoenmakers, A. P., Mack, K. H., de Bruyn, A. G., et al., 2000b, A\&AS, 146, 293

Schoenmakers, A. P, de Bruyn, A. G., Röttgering, H. J. A., \& van der Laan, H., 2001, A\&A, 374, 861

Sebastian, B., Ishwara-Chandra, C. H., Joshi, R., \& Wadadekar, Y., 2018, MNRAS, 473, 4926

Simpson, C., Martinez-Sansigre, A., Rawlings, S., et al., 2006, MNRAS, 372, 741

Singal, A. K., 1988, MNRAS, 233, 87

Singal, A. K., 1996, IAUS, 175, 563

Singal, A. K., Konar, C., \& Saikia, D. J., 2004, MNRAS, 347, 79

Skrutskie, M.F., Cutri, R.M., Stiening, R., et al. 2006, AJ, 131, 1163

Solovyov, D. I., \& Verkhodanov, O. V., 2014, AstBu, 69, 141

Subrahmanyan, R., Saripalli, L., \& Hunstead, R. W., 1996, MNRAS, 279, 257

Subrahmanyan , R., Saripalli, L., Safouris, V., \& Hunstead, R. W., 2008, ApJ, 677, 63

Subrahmanyan, R., Ekers, R. D., Saripalli, L., \& Sadler, E. M., 2010, MNRAS, 402, 2792

Tamhane, P., Wadadekar, Y., Basu, A., et al., 2015, MNRAS, 453, 2438

van Breugel W. J. M., \& Willis A. G., 1981, A\&A, 96, 332

van Breugel, W. J. M., \& Jägers, W., 1982, A\&AS, 49, 529

van Haarlem, M. P., Wise, M. W.,Gunst, A. W., et al. 2013, A\&A, 556,2

Weżgowiec, M., Jamrozy, M., \& Mack, K.-H., 2016, AcA, 66, 85

Willis, A. G., Strom, R. G., \& Wilson, A. S., 1974, Nature, 250, 625

Wing, J. D., \& Blanton, E. L., 2011, AJ, 141, 88

Winkler, C., 1994, ApJS, 92, 327

Wright, E. L., Eisenhardt, P. R. M., Mainzer, A. K., et al., 2010, AJ, 140, 1868 
Table 1: List of GRSs

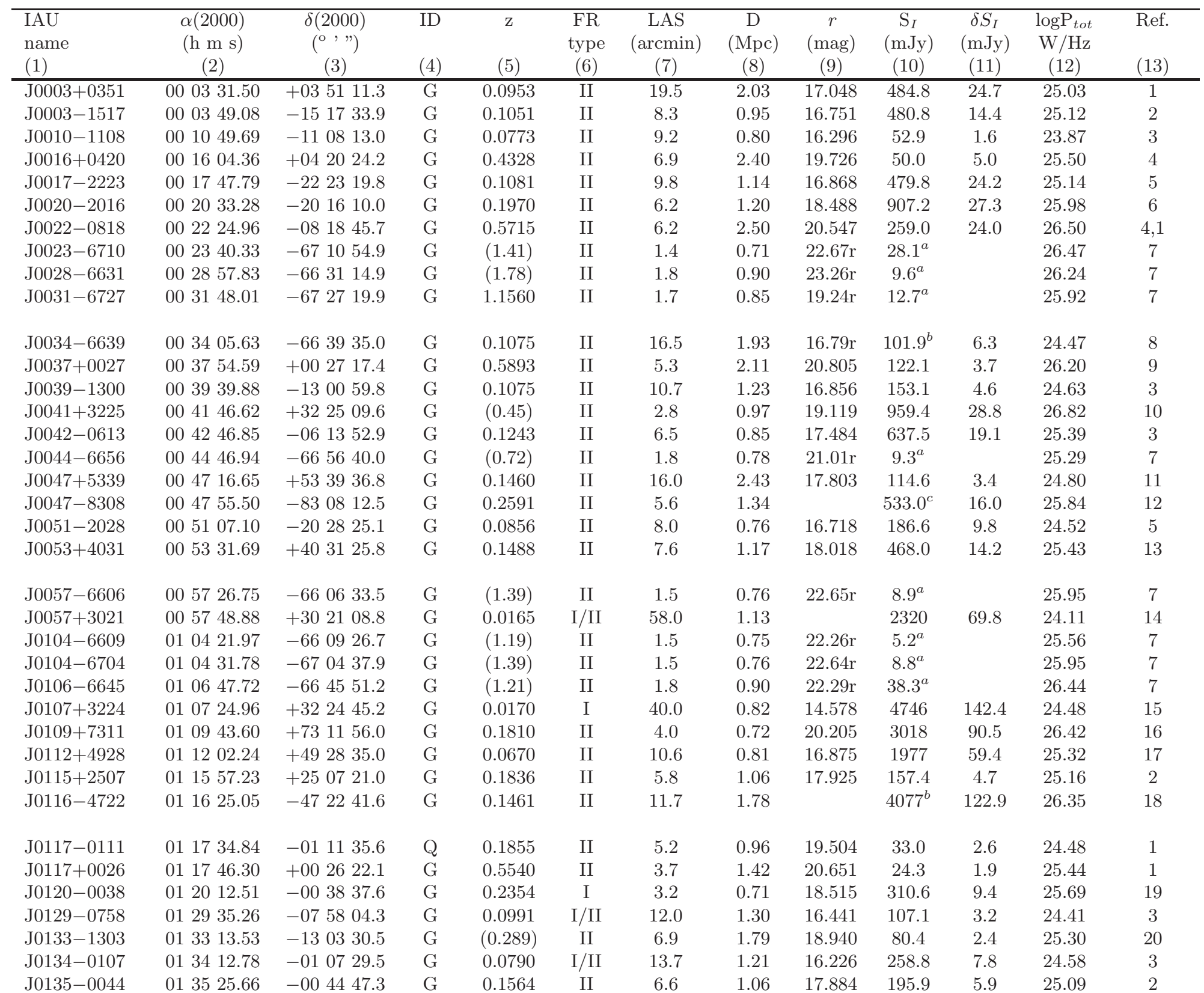




\begin{tabular}{|c|c|c|c|c|c|c|c|c|c|c|c|c|}
\hline $\begin{array}{l}\text { IAU } \\
\text { name } \\
(1)\end{array}$ & $\begin{array}{c}\alpha(2000) \\
(\mathrm{h} \mathrm{m} \mathrm{s}) \\
(2)\end{array}$ & $\begin{array}{c}\delta(2000) \\
\left(\begin{array}{c}0 \\
(3)\end{array}\right) \\
(3)\end{array}$ & (4) & (5) & $\begin{array}{c}\text { FR } \\
\text { type } \\
(6)\end{array}$ & $\begin{array}{c}\text { LAS } \\
(\operatorname{arcmin}) \\
(7)\end{array}$ & $\begin{array}{c}\mathrm{D} \\
(\mathrm{Mpc}) \\
(8)\end{array}$ & $\begin{array}{c}r \\
(\mathrm{mag}) \\
(9)\end{array}$ & $\begin{array}{c}\mathrm{S}_{I} \\
(\mathrm{mJy}) \\
(10)\end{array}$ & $\begin{array}{c}\delta S_{I} \\
(\mathrm{mJy}) \\
(11)\end{array}$ & $\begin{array}{c}\log \mathrm{P}_{t o t} \\
\mathrm{~W} / \mathrm{Hz} \\
(12)\end{array}$ & $\begin{array}{l}\text { Ref. } \\
\text { (13) }\end{array}$ \\
\hline J0135+3754 & 013528.33 & +375405.6 & $\mathrm{G}$ & 0.4373 & II & 2.7 & 0.91 & 19.422 & 1331 & 40.0 & 26.93 & 21 \\
\hline J0143-5431 & 014343.13 & -543139.3 & G & 0.1791 & II & 5.5 & 0.99 & & $217.0^{c}$ & 6.5 & 25.09 & 12 \\
\hline J0152+0015 & $0152 \quad 14.26$ & +00 1502.6 & G & 0.8440 & II & 2.2 & 1.03 & 20.818 & 111.6 & 3.4 & 26.53 & 23 \\
\hline J0155-2654 & 015546.30 & -265404.7 & G & 0.2091 & II & 3.8 & 0.77 & 17.843 & 22.3 & 0.7 & 24.43 & 24 \\
\hline J0202-0939 & 020224.09 & -09 3900.3 & G & 0.7683 & II & 2.6 & 1.12 & 20.948 & 433.7 & 21.7 & 27.02 & 1,3 \\
\hline J0204-0944 & 020448.29 & -094409.5 & Q & 1.0033 & II & 4.2 & 2.08 & 18.904 & 13.6 & 0.4 & 25.80 & 25 \\
\hline $\mathrm{J} 0210+0118$ & $02 \quad 1008.48$ & +01 1839.6 & Q & 0.8652 & II & 2.6 & 1.21 & 18.595 & 33.1 & 1.0 & 26.03 & 25 \\
\hline J0213-4744 & 021309.53 & -474413.4 & G & 0.2200 & II & 6.3 & 1.33 & & $2115^{b}$ & 64.3 & 26.46 & 18 \\
\hline J0214+3251 & $02 \quad 14 \quad 15.38$ & +32 5105.3 & G & 0.2610 & II & 5.2 & 1.25 & 19.068 & 451.1 & 13.6 & 25.95 & 26 \\
\hline J0216-0449 & $02 \quad 16 \quad 59.24$ & -044920.3 & G & 1.3250 & II & 2.4 & 1.20 & & 9.6 & 0.3 & 25.94 & 27,28 \\
\hline J0300-0728 & 030059.06 & -072830.8 & G & 0.4905 & II & 5.0 & 1.81 & 20.611 & 71.9 & 2.2 & 25.78 & 3 \\
\hline $\mathrm{J} 0313+4120$ & 031301.96 & +412001.2 & G & 0.1340 & I/II & 9.5 & 1.34 & 17.359 & 489.8 & 14.8 & 25.35 & 30 \\
\hline J0313-0631 & 031332.88 & -063157.9 & Q & 0.3889 & II & 3.5 & 1.11 & 19.325 & 151.8 & 4.6 & 25.87 & 3 \\
\hline J0315-0743 & $03 \quad 1536.19$ & -074338.8 & G & $(0.269)$ & II & 3.3 & 0.86 & 19.140 & 151.0 & 16.0 & 25.50 & 4 \\
\hline J0316-2658 & $03 \quad 16 \quad 04.24$ & -265806.0 & G & 0.2171 & II & 3.8 & 0.79 & 17.466 & 453.5 & 13.8 & 25.77 & 6 \\
\hline J0318+6829 & 031818.98 & +68 2931.4 & G & 0.0901 & II & 14.9 & 1.48 & 18.650 & 777.5 & 23.5 & 25.18 & 31 \\
\hline J0320-4515 & 032057.54 & $-45 \quad 1510.7$ & G & 0.0630 & II & 25.6 & 1.84 & & $6657^{b}$ & 201.7 & 25.79 & 32 \\
\hline J0326-7730 & 032601.33 & -773016.0 & $\mathrm{G}$ & 0.2771 & II & 5.3 & 1.33 & & $357.0^{c}$ & 10.7 & 25.73 & 12 \\
\hline J0331-7713 & 033139.79 & $\begin{array}{lll}-77 & 13 & 19.2\end{array}$ & G & 0.1456 & II & 17.7 & 2.69 & & $687.0^{c}$ & 20.6 & 25.40 & 12 \\
\hline $\mathrm{J} 0349+7511$ & $03 \quad 49 \quad 16.28$ & +751122.0 & G & 0.0803 & II & 14.5 & 1.30 & 16.609 & 30 & 0.9 & 23.66 & 33 \\
\hline J0351-1429 & 035128.54 & -142908.7 & Q & 0.6163 & II & 2.1 & 0.84 & 15.890 & 3049 & 91.5 & 27.65 & 34 \\
\hline J0401-8456 & 040118.32 & -845636.1 & G & 0.1037 & $\mathrm{I}$ & 6.3 & 0.71 & & $175.0^{c}$ & 5.3 & 24.49 & 12 \\
\hline $\mathrm{J} 0449+4500$ & 044909.08 & +450039.2 & G & 0.0208 & $\mathrm{I}$ & 30.0 & 0.76 & 17.858 & 6502 & 195.1 & 24.80 & 40 \\
\hline J0449-3026 & 044932.35 & -302637.7 & Q & 0.3149 & II & 7.5 & 2.14 & 17.945 & 94.0 & 9.0 & 25.45 & 4 \\
\hline
\end{tabular}




\begin{tabular}{|c|c|c|c|c|c|c|c|c|c|c|c|c|}
\hline $\begin{array}{l}\text { IAU } \\
\text { name } \\
(1)\end{array}$ & $\begin{array}{c}\alpha(2000) \\
(\mathrm{h} \mathrm{m} \mathrm{s}) \\
(2)\end{array}$ & $\begin{array}{c}(2000) \\
\left(\begin{array}{c}\circ \\
(3)\end{array}\right) \\
(3)\end{array}$ & (4) & (5) & $\begin{array}{l}\text { FR } \\
\text { type } \\
(6)\end{array}$ & $\begin{array}{c}\text { LAS } \\
(\operatorname{arcmin}) \\
(7)\end{array}$ & $\begin{array}{c}\mathrm{D} \\
(\mathrm{Mpc}) \\
(8)\end{array}$ & $\begin{array}{c}r \\
(\mathrm{mag}) \\
(9)\end{array}$ & $\begin{array}{c}\mathrm{S}_{I} \\
(\mathrm{mJy}) \\
(10)\end{array}$ & $\begin{array}{c}\delta S_{I} \\
(\mathrm{mJy}) \\
(11)\end{array}$ & $\begin{array}{c}\log \mathrm{P}_{t o t} \\
\mathrm{~W} / \mathrm{Hz} \\
(12)\end{array}$ & $\begin{array}{l}\text { Ref. } \\
\text { (13) }\end{array}$ \\
\hline J0452+5204 & 045252.84 & +520447.1 & $\mathrm{G}$ & 0.1090 & I & 9.7 & 1.15 & 17.530 & 2853 & 85.6 & 25.92 & 40 \\
\hline J0459-5250 & $04 \quad 5916.33$ & -525008.1 & $\mathrm{G}$ & 0.0959 & II & 9 & 0.90 & & $7.3^{b}$ & 1.0 & 23.04 & 41 \\
\hline J0505-2835 & $05 \quad 05 \quad 49.22$ & -283519.4 & G & 0.0381 & II & 40.0 & 1.79 & & 713.9 & 21.8 & 24.37 & 42 \\
\hline J0508+6056 & $05 \quad 08 \quad 27.26$ & +605627.3 & G & 0.0710 & $\mathrm{I}$ & 10.4 & 0.84 & 17.638 & 189.7 & 6.1 & 24.35 & 37 \\
\hline J0513-3028 & 051331.98 & -302850.1 & G & 0.0576 & II & 11.4 & 0.76 & 15.987 & 2279 & 68.4 & 25.25 & 18 \\
\hline J0515-8059 & 051555.18 & -80 5943.1 & G & 0.1049 & $\mathrm{I} / \mathrm{II}$ & 7.5 & 0.86 & & $267.0^{c}$ & 8.0 & 24.68 & 12 \\
\hline J0607+6114 & 060734.92 & +611443.5 & G & 0.2270 & I/II & 5.3 & 1.15 & 18.895 & 346.4 & 10.6 & 25.70 & 37 \\
\hline J0632-5404 & 063201.17 & -540457.5 & Q & 0.2036 & II & 5.2 & 1.04 & & $694.0^{c}$ & 20.8 & 25.72 & 12 \\
\hline J0636-2034 & 063632.26 & -203453.2 & G & 0.0552 & II & 15.0 & 0.95 & 16.267 & 8485 & 254.6 & 25.77 & 43 \\
\hline J0652+4304 & 065218.38 & +430459.7 & G & 0.0891 & II & 9.6 & 0.95 & 16.678 & 315.7 & 9.8 & 24.78 & 26 \\
\hline J0654+7319 & 065426.69 & +731950.2 & G & 0.1145 & II & 12.6 & 1.56 & 18.644 & 828.6 & 24.9 & 25.43 & 26 \\
\hline J0657+4808 & 065751.78 & +480829.7 & G & 0.7760 & II & 2.2 & 0.98 & 20.697 & 78.2 & 2.6 & 26.29 & 44 \\
\hline J0725+3025 & $07 \quad 2517.39$ & +302536.3 & G & $(0.70)$ & II & 2.9 & 1.24 & 19.745 & 31.0 & 1.5 & 25.78 & 46 \\
\hline $\mathrm{J} 0745+0200$ & 074504.47 & +020008.1 & G & 0.4650 & II & 2.1 & 0.74 & 20.220 & 1535 & 46.0 & 27.06 & 47 \\
\hline J0746-5702 & 074618.62 & -570258.2 & G & 0.1300 & $\mathrm{I}$ & 7.4 & 1.02 & & $194.0^{c}$ & 5.8 & 24.74 & 12 \\
\hline J0748+5548 & 074836.87 & +554858.3 & G & 0.0356 & II & 34.0 & 1.44 & 15.806 & 1822 & 54.7 & 24.73 & 14 \\
\hline J0750+6541 & 075034.40 & +654125.6 & $\mathrm{Q}$ & 0.7490 & II & 3.7 & 1.63 & 17.702 & 116.7 & 3.8 & 26.43 & 37 \\
\hline J0751+4231 & 075108.80 & +423124.2 & $\mathrm{G}$ & 0.2042 & II & 6.0 & 1.19 & 18.142 & 138.3 & 4.5 & 25.19 & 26 \\
\hline J0754+4316 & 075407.96 & +431610.6 & Q & 0.3476 & II & 8.1 & 2.37 & 16.722 & 101.9 & 3.5 & 25.59 & 25,26 \\
\hline J0754+3033 & 075448.85 & +303355.0 & $\mathrm{Q}$ & 0.7961 & II & 3.8 & 1.71 & 17.727 & 60.7 & 1.8 & 26.21 & 25 \\
\hline $\mathrm{J} 0801+4736$ & 080131.97 & +473616.1 & Q & 0.1567 & II & 6.0 & 0.97 & 15.642 & 124.9 & 4.0 & 24.90 & 25,26 \\
\hline J0802+4927 & 080248.80 & +492723.8 & G & $(0.678)$ & II & 4.3 & 1.88 & 21.317 & 52.0 & 7.0 & 25.98 & 4 \\
\hline J0803+6656 & 080345.83 & +665611.4 & G & 0.2470 & II & 4.7 & 1.08 & 20.629 & 174.0 & 5.5 & 25.48 & 37 \\
\hline $\mathrm{J} 0807+7400$ & $0807 \quad 10.16$ & +740041.8 & G & 0.1204 & II & 9.1 & 1.17 & 17.175 & 128.8 & 4.4 & 24.67 & 26 \\
\hline J0826+6920 & 082601.01 & +692037.0 & G & 0.5380 & II & 6.7 & 2.54 & 21.133 & 238.8 & 4.6 & 26.40 & 49 \\
\hline $\mathrm{J} 0842+2147$ & 084239.95 & +214710.3 & $\mathrm{Q}$ & 1.1814 & II & 2.2 & 1.08 & 18.948 & 44.9 & 1.3 & 26.49 & 48 \\
\hline
\end{tabular}




\begin{tabular}{|c|c|c|c|c|c|c|c|c|c|c|c|c|}
\hline $\begin{array}{l}\text { IAU } \\
\text { name } \\
(1)\end{array}$ & $\begin{array}{c}\alpha(2000) \\
(\mathrm{h} \mathrm{m} \mathrm{s}) \\
(2)\end{array}$ & $\begin{array}{c}\delta(2000) \\
\left(\begin{array}{c}0 \\
(3)\end{array}\right) \\
(3)\end{array}$ & (4) & (5) & $\begin{array}{c}\text { FR } \\
\text { type } \\
(6)\end{array}$ & $\begin{array}{c}\text { LAS } \\
(\operatorname{arcmin}) \\
(7)\end{array}$ & $\begin{array}{c}\mathrm{D} \\
(\mathrm{Mpc}) \\
(8)\end{array}$ & $\begin{array}{c}r \\
(\mathrm{mag}) \\
(9)\end{array}$ & $\begin{array}{c}\mathrm{S}_{I} \\
(\mathrm{mJy}) \\
(10)\end{array}$ & $\begin{array}{c}\delta S_{I} \\
(\mathrm{mJy}) \\
(11)\end{array}$ & $\begin{array}{c}\log \mathrm{P}_{t o t} \\
\mathrm{~W} / \mathrm{Hz} \\
(12)\end{array}$ & $\begin{array}{l}\text { Ref. } \\
\text { (13) }\end{array}$ \\
\hline J0843-7006 & $08 \quad 4305.41$ & -700656.1 & $\mathrm{G}$ & 0.1393 & I & 6.9 & 1.01 & 17.651 & $203.0^{c}$ & 6.1 & 24.82 & 12 \\
\hline J0844+4627 & 084408.85 & +462744.2 & G & 0.5697 & II & 5.5 & 2.14 & 20.816 & $82.3^{d}$ & 2.5 & 25.49 & 51 \\
\hline J0856+6621 & $\begin{array}{lll}08 & 56 & 16.32\end{array}$ & +66 2126.8 & G & 0.4890 & II & 3.8 & 1.37 & 20.289 & 216.4 & 6.6 & 26.26 & 37 \\
\hline J0857+0131 & 085701.76 & +013130.9 & G & 0.2734 & II & 5.2 & 1.30 & 18.953 & 99.0 & 9.0 & 25.34 & 4 \\
\hline J0857+3945 & 085743.54 & +394528.7 & G & 0.5288 & II & 2.8 & 1.05 & 19.962 & 501.8 & 15.1 & 26.70 & 21 \\
\hline J0902+5707 & 090207.20 & +570737.9 & Q & 1.5964 & II & 1.7 & 0.87 & 18.749 & 29.3 & 0.9 & 26.61 & 52 \\
\hline J0902+1737 & 090238.42 & +173751.5 & G & 0.1645 & II & 7.1 & 1.19 & 17.749 & 129.5 & 3.9 & 24.96 & 2 \\
\hline J0903+1208 & $0903 \quad 03.53$ & +120858.6 & G & 0.3444 & II & 5.3 & 1.54 & 19.267 & 107.9 & 3.2 & 25.60 & 3 \\
\hline J0908+3942 & $0908 \quad 18.57$ & +394257.1 & G & 1.8830 & II & 1.9 & 0.97 & 21.098 & 259.2 & 7.9 & 27.73 & 53 \\
\hline J0908+3506 & $0908 \quad 47.89$ & +350621.9 & G & 0.2600 & II & 6.3 & 1.51 & 18.483 & 196.6 & 6.1 & 25.59 & 26 \\
\hline J0912+3510 & $09 \quad 1251.67$ & +351012.0 & G & 0.2489 & II & 6.3 & 1.46 & 19.374 & 153.2 & 2.9 & 25.44 & 45 \\
\hline J0925-0114 & 092512.73 & -011441.3 & G & 0.0730 & II & 13.9 & 1.14 & 16.344 & 82.5 & 2.5 & 24.02 & 3 \\
\hline J0925+4004 & 092554.72 & +4004 14.2 & Q & 0.4717 & II & 4.4 & 1.55 & 17.891 & 75.5 & 2.3 & 25.76 & 25 \\
\hline J0926+6519 & 092600.82 & +65 1922.7 & G & 0.1397 & $\mathrm{I}$ & 5.3 & 0.78 & 17.125 & 300.9 & 9.2 & 25.18 & 37 \\
\hline J0926+6100 & 092653.38 & +610025.2 & Q & 0.2430 & II & 3.7 & 0.84 & 18.758 & 93.2 & 3.3 & 25.20 & 37 \\
\hline J0927+3511 & 092749.38 & +351104.2 & $\mathrm{G}$ & $(0.55)$ & II & 5.8 & 2.23 & 17.455 & 83.4 & 2.9 & 25.96 & 46 \\
\hline J0929+4146 & 092910.67 & +414645.5 & $\mathrm{G}$ & 0.3650 & II & 6.6 & 2.00 & 19.792 & 164.6 & 5.4 & 25.84 & 26 \\
\hline J0931+3204 & 093139.05 & +320400.1 & $\mathrm{Q}$ & 0.2267 & II & 19.9 & 4.29 & 17.487 & 65.6 & 2.0 & 24.97 & 50 \\
\hline J0932+1611 & 093238.30 & +161157.2 & G & 0.1910 & II & 4.0 & 0.76 & 17.817 & 746.3 & 22.5 & 25.87 & 54 \\
\hline J0937+2937 & 093704.04 & +293704.8 & Q & 0.4513 & II & 2.6 & 0.90 & 17.699 & 28.9 & 0.9 & 25.30 & 25 \\
\hline J0939+7405 & 093947.07 & +740529.8 & G & 0.1215 & II & 7.3 & 0.95 & 16.765 & 87.6 & 3.3 & 24.51 & 26 \\
\hline J0939+3553 & 093952.76 & +355358.9 & G & 0.1367 & II & 5.4 & 0.78 & 17.444 & 3542 & 106.3 & 26.23 & 55 \\
\hline $\mathrm{J} 1006+3454$ & 100601.74 & +345410.4 & G & 0.0994 & II & 39.0 & 4.23 & 16.759 & 4481 & 134.5 & 26.03 & 21 \\
\hline $\mathrm{J} 1011+3111$ & 101112.11 & +311104.5 & G & $(0.50)$ & II & 4.8 & 1.75 & 21.556 & 57.3 & 2.1 & 25.70 & 46 \\
\hline
\end{tabular}

$\mathrm{J} 1011+311$

(0.50) 


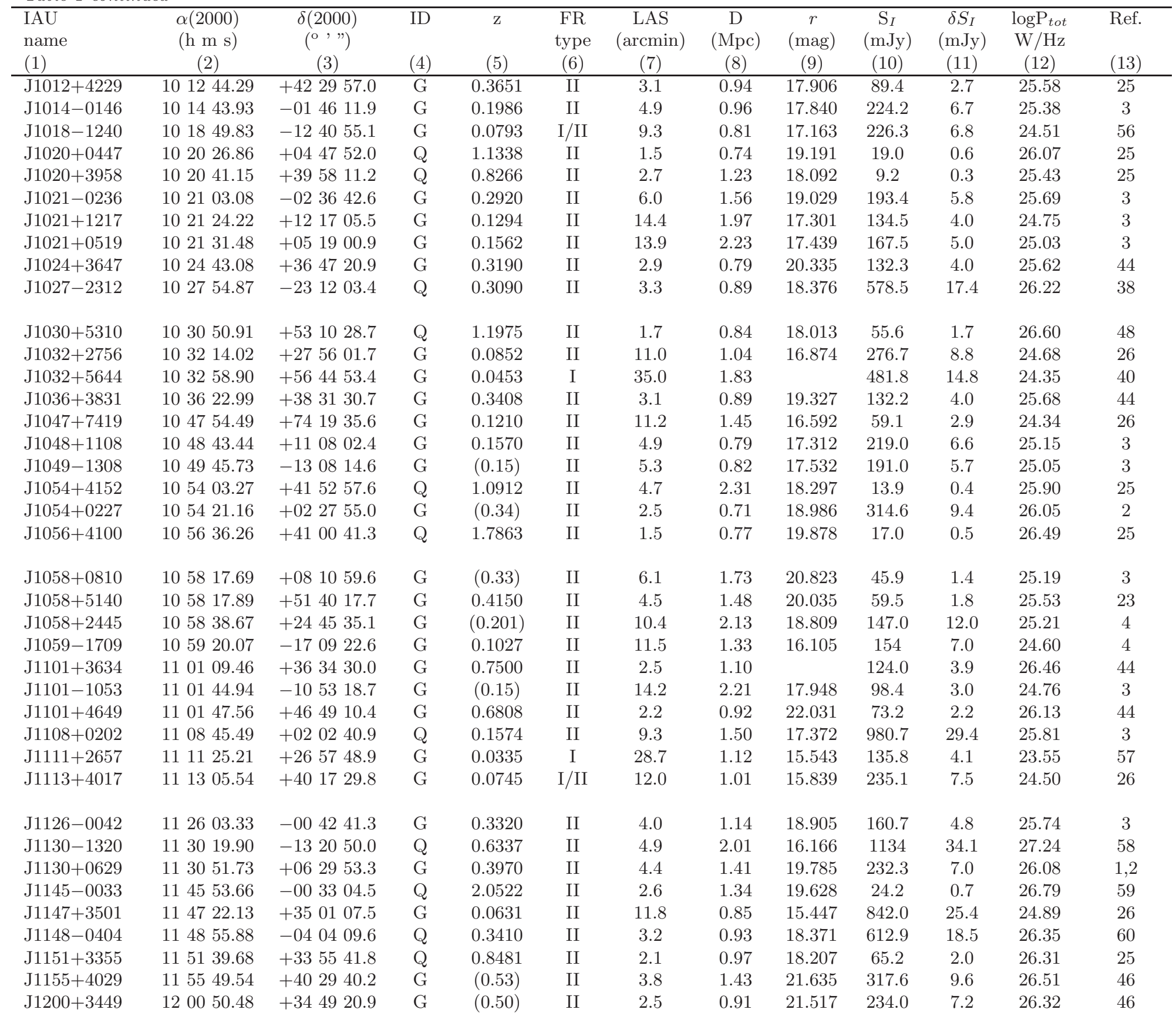




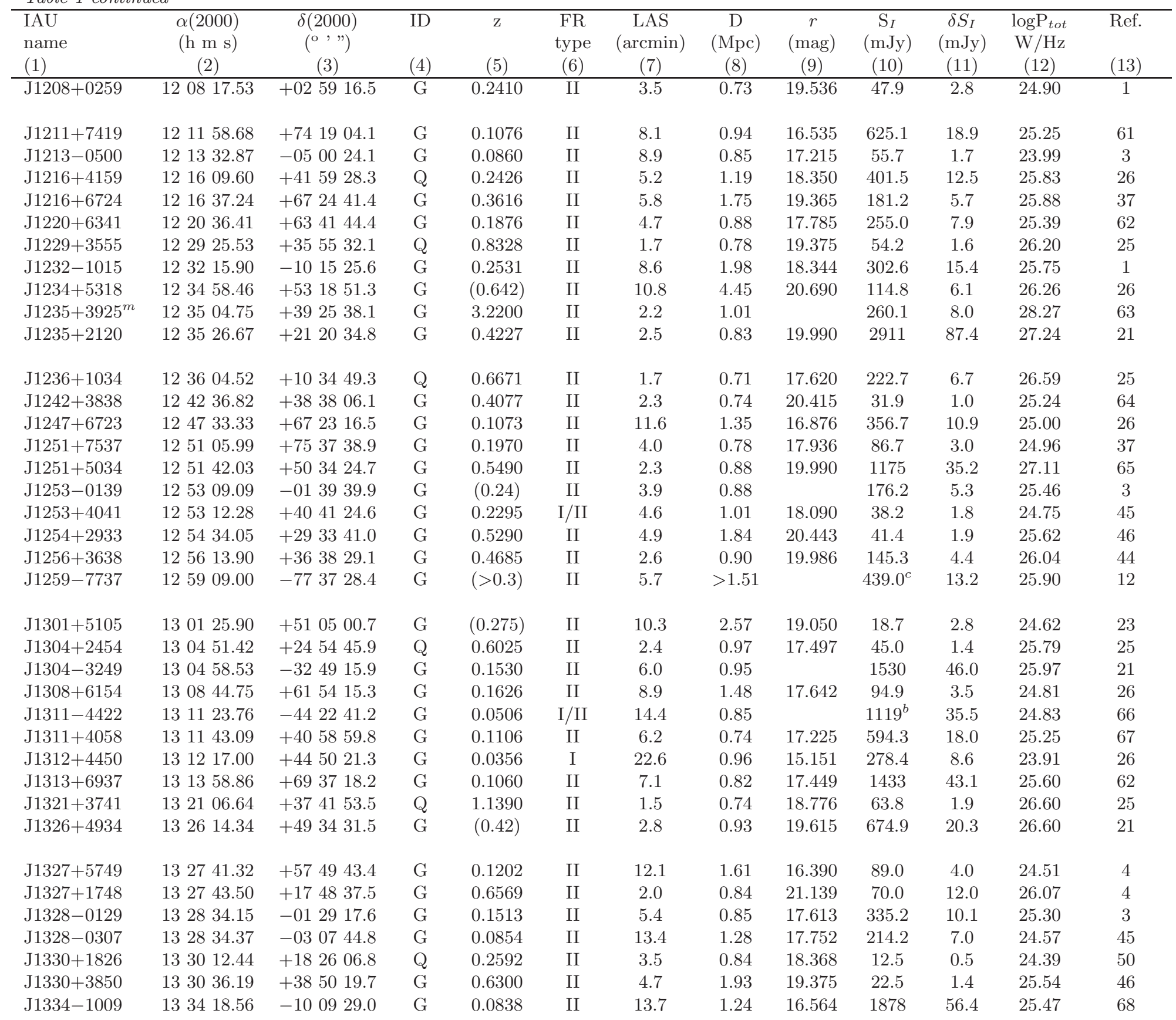




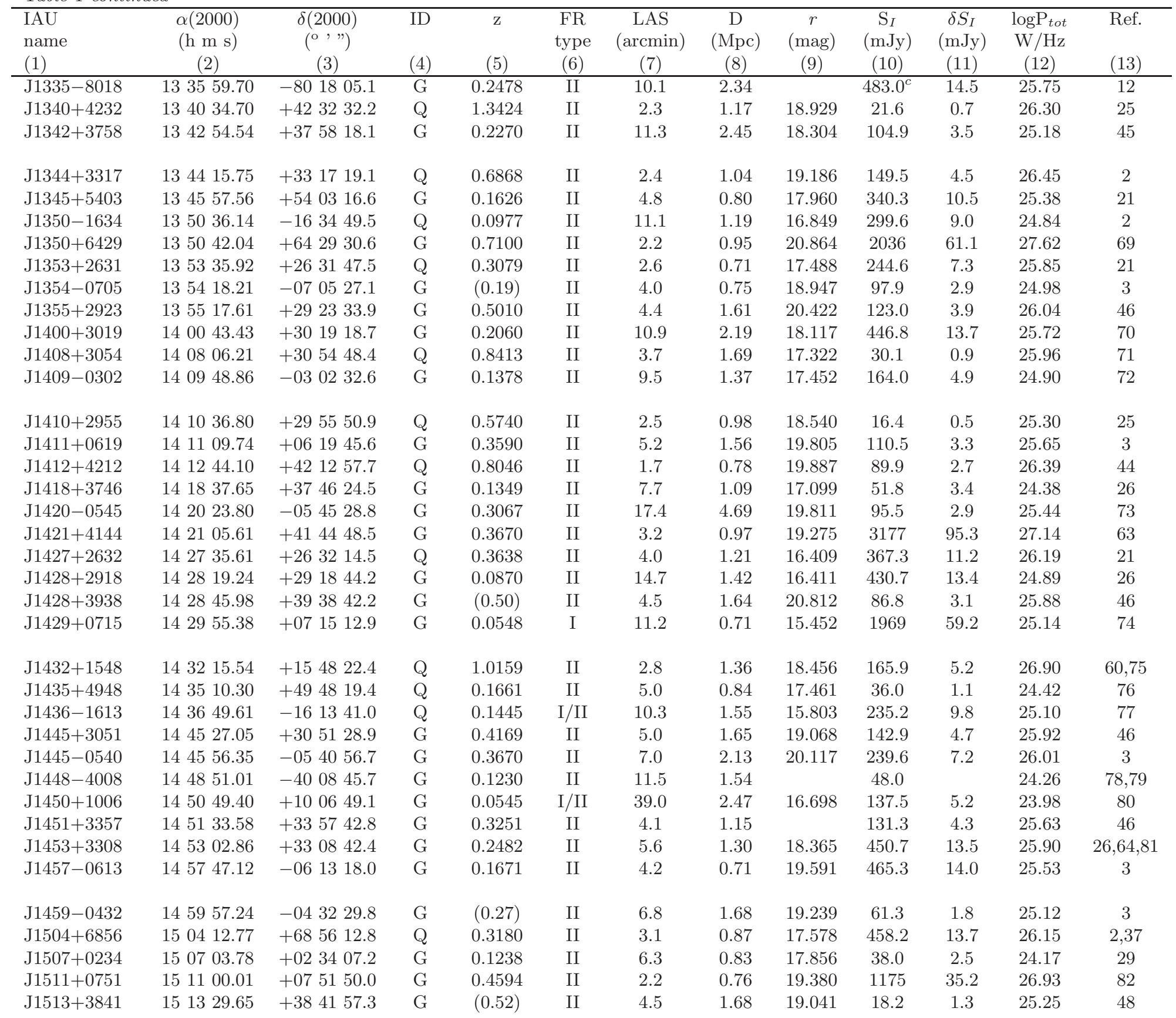




\begin{tabular}{|c|c|c|c|c|c|c|c|c|c|c|c|c|}
\hline $\begin{array}{l}\text { IAU } \\
\text { name } \\
(1) \\
\end{array}$ & $\begin{array}{c}\alpha(2000) \\
(\mathrm{h} \mathrm{m} \mathrm{s}) \\
(2) \\
\end{array}$ & $\begin{array}{c}\delta(2000) \\
\left(\begin{array}{c}{ }^{\circ}, " \\
\end{array}\right) \\
(3) \\
\end{array}$ & $(4)$ & $\begin{array}{l}\mathrm{Z} \\
(5) \\
\end{array}$ & $\begin{array}{c}\text { FR } \\
\text { type } \\
(6) \\
\end{array}$ & $\begin{array}{c}\text { LAS } \\
\left(\begin{array}{c}\operatorname{arcmin}) \\
(7)\end{array}\right. \\
\end{array}$ & $\begin{array}{c}\mathrm{D} \\
(\mathrm{Mpc}) \\
(8) \\
\end{array}$ & $\begin{array}{c}r \\
(\mathrm{mag}) \\
(9) \\
\end{array}$ & $\begin{array}{c}\mathrm{S}_{I} \\
(\mathrm{mJy}) \\
(10) \\
\end{array}$ & $\begin{array}{c}\delta S_{I} \\
(\mathrm{mJy}) \\
(11) \\
\end{array}$ & $\begin{array}{c}\log \mathrm{P}_{t o t} \\
\mathrm{~W} / \mathrm{Hz} \\
(12) \\
\end{array}$ & $\begin{array}{l}\text { Ref. } \\
\text { (13) } \\
\end{array}$ \\
\hline J1520-0546 & 152013.29 & -054627.0 & $\mathrm{G}$ & 0.0605 & II & 22.0 & 1.53 & 16.485 & 26.8 & 0.8 & 23.37 & 3 \\
\hline $\mathrm{J} 1525+3345$ & 152500.79 & +334542.4 & G & $(0.47)$ & II & 3.6 & 1.27 & 20.974 & 44.9 & 2.0 & 25.54 & 46 \\
\hline $\mathrm{J} 1536+8423$ & 153657.27 & +842310.8 & $\mathrm{G}$ & 0.2010 & II & 7.9 & 1.56 & 18.989 & 368.0 & 11.3 & 25.61 & 26 \\
\hline J1540-0127 & 154056.80 & -012710.0 & $\mathrm{G}$ & 0.1490 & II & 4.9 & 0.76 & 17.679 & 194.1 & 5.8 & 25.05 & 3 \\
\hline $\mathrm{J} 1552+2005$ & 155209.19 & +200523.2 & G & 0.0890 & II & 19.6 & 1.95 & 17.353 & 2384 & 71.6 & 25.67 & 14 \\
\hline J1554+3945 & 155426.85 & +394508.7 & $\mathrm{G}$ & $(0.35)$ & II & 3.7 & 1.09 & 19.997 & 69.3 & 2.5 & 25.43 & 46 \\
\hline $\mathrm{J} 1555+3653$ & 155500.39 & +365337.4 & G & 0.2472 & II & 5.8 & 1.34 & 18.891 & 100.9 & 3.5 & 25.25 & 46 \\
\hline J1604+3731 & 160423.44 & +373149.3 & $\mathrm{G}$ & 0.8140 & II & 2.9 & 1.32 & & 117.0 & 3.8 & 26.52 & 44 \\
\hline J1604+3438 & 160445.89 & +343816.5 & G & 0.2817 & II & 3.3 & 0.84 & 19.611 & 139.6 & 4.5 & 25.52 & 46 \\
\hline $\mathrm{J} 1615+3826$ & $16 \quad 15 \quad 52.23$ & +382631.9 & $\mathrm{G}$ & 0.1856 & II & 4.4 & 0.81 & 17.942 & 27.0 & 1.5 & 24.40 & 46 \\
\hline $\mathrm{J} 1635+3608$ & 163522.54 & +360804.9 & G & 0.1650 & $\mathrm{I} / \mathrm{II}$ & 5.3 & 0.90 & 17.731 & 95.1 & 3.3 & 24.83 & 46 \\
\hline $\mathrm{J} 1637+4146$ & 163753.38 & +414601.4 & G & 0.8670 & II & 2.2 & 1.02 & & 64.7 & 2.3 & 26.33 & 44 \\
\hline $\mathrm{J} 1649+3114$ & 164906.12 & +311431.4 & G & 0.4373 & II & 3.5 & 1.18 & 19.261 & 146.8 & 4.7 & 25.98 & 46 \\
\hline $\mathrm{J} 1702+4217$ & 170255.95 & +421748.8 & $\mathrm{G}$ & 0.4760 & II & 3.0 & 1.07 & 21.780 & 181.6 & 5.7 & 26.16 & 44 \\
\hline $\mathrm{J} 1706+4340$ & 170625.44 & +434040.2 & $\mathrm{Q} / \mathrm{G}$ & $(0.525)$ & II & 2.0 & 0.75 & 19.743 & 148.0 & 4.4 & 26.17 & 84 \\
\hline $\mathrm{J} 1712+3558$ & 171224.87 & +355826.2 & G & 0.3357 & II & 3.5 & 1.00 & 20.025 & 82.6 & 2.9 & 25.46 & 46 \\
\hline $\mathrm{J} 1723+3417$ & 172320.80 & +341758.0 & $\mathrm{Q}$ & 0.2060 & II & 4.1 & 0.82 & 16.043 & 1639 & 49.2 & 26.28 & 38 \\
\hline $\mathrm{J} 1725+3923$ & 172517.05 & +392305.3 & G & 0.2898 & II & 4.8 & 1.24 & 22.008 & 78.8 & 3.0 & 25.30 & 46 \\
\hline J1728-7237 & 172828.10 & -723734.9 & G & 0.4735 & II & 6.2 & 2.20 & & $214.0^{c}$ & 6.4 & 26.05 & 12 \\
\hline $\mathrm{J} 1738+3733$ & 173820.93 & +373333.8 & G & 0.1562 & II & 6.5 & 1.04 & 17.419 & 235.5 & 7.3 & 25.17 & 26 \\
\hline $\mathrm{J} 1745+7115$ & 174543.53 & +711548.7 & G & 0.2160 & II & 4.4 & 0.92 & 18.805 & 887.5 & 26.7 & 26.06 & 37 \\
\hline J1748-2335 & 174839.05 & -233521.2 & G & 0.2400 & II & 5.7 & 1.29 & & 398.5 & 12.0 & 25.81 & 78,85 \\
\hline J1853+8002 & 185351.07 & +800242.5 & $\mathrm{G}$ & 0.2139 & II & 5.6 & 1.16 & 19.994 & 153.9 & 5.0 & 25.29 & 37 \\
\hline J1910-7049 & 191055.20 & -704900.9 & G & 0.2152 & II & 6.0 & 1.25 & & $238.0^{c}$ & 7.1 & 25.31 & 12 \\
\hline J1918+7415 & 191834.89 & +741505.1 & G & 0.1940 & II & 6.6 & 1.26 & 18.179 & 571.7 & 17.3 & 25.77 & 37 \\
\hline
\end{tabular}




\begin{tabular}{|c|c|c|c|c|c|c|c|c|c|c|c|c|}
\hline $\begin{array}{l}\text { IAU } \\
\text { name } \\
(1)\end{array}$ & $\begin{array}{c}\alpha(2000) \\
(\mathrm{h} \mathrm{m} \mathrm{s}) \\
(2)\end{array}$ & $\begin{array}{c}\delta(2000) \\
\left(\begin{array}{c}\mathrm{o} \\
, "\end{array}\right) \\
(3)\end{array}$ & (4) & $(5)$ & $\begin{array}{l}\text { FR } \\
\text { type } \\
(6)\end{array}$ & $\begin{array}{c}\text { LAS } \\
(\operatorname{arcmin}) \\
(7)\end{array}$ & $\begin{array}{c}\mathrm{D} \\
(\mathrm{Mpc}) \\
(8)\end{array}$ & $\begin{array}{c}r \\
(\mathrm{mag}) \\
(9)\end{array}$ & $\begin{array}{c}\mathrm{S}_{I} \\
(\mathrm{mJy}) \\
(10)\end{array}$ & $\begin{array}{c}\delta S_{I} \\
(\mathrm{mJy}) \\
(11)\end{array}$ & $\begin{array}{c}\log \mathrm{P}_{t o t} \\
\mathrm{~W} / \mathrm{Hz} \\
(12) \\
\end{array}$ & Ref. \\
\hline J1919-7959 & 191913.86 & -795906.7 & $G$ & 0.3460 & II & 6.1 & 1.78 & 19.622 & $1262^{c}$ & 37.9 & 26.50 & 18 \\
\hline $\mathrm{J} 1920+4526$ & 192001.66 & +452652.9 & $\mathrm{G}$ & 0.0522 & II & 16.7 & 1.01 & 15.340 & 287.9 & 14.9 & 24.26 & 26 \\
\hline $\mathrm{J} 1921+4806$ & $1921 \quad 13.97$ & +480618.7 & G & 0.1023 & $\mathrm{I}$ & 13.5 & 1.52 & 16.489 & 1032 & 51.7 & 25.42 & 26 \\
\hline J1946-8222 & 194650.50 & -822253.8 & G & 0.3330 & II & 7.4 & 2.11 & & $219.0^{c}$ & 6.6 & 25.70 & 12 \\
\hline $\mathrm{J} 1951+7037$ & 195140.82 & +703740.0 & G & 0.5500 & II & 5.2 & 2.00 & 20.825 & 92.3 & 3.2 & 26.01 & 37 \\
\hline J2018-5539 & 201801.31 & -553930.8 & G & 0.0606 & I & 20 & 1.37 & & $2653^{b}$ & 80.9 & 25.35 & 66,90 \\
\hline J2034-2630 & 203449.23 & -263036.4 & G & 0.1033 & II & 6.7 & 0.78 & 16.547 & 76.0 & 6.0 & 24.30 & 4 \\
\hline $\mathrm{J} 2035+6805$ & 203516.55 & +680541.6 & G & 0.1330 & I & 11.5 & 1.61 & 18.568 & 253.7 & 8.1 & 25.06 & 37 \\
\hline $\mathrm{J} 2042+7508$ & 204237.31 & +750802.4 & Q & 0.1040 & II & 10.2 & 1.16 & 14.576 & 1789 & 53.7 & 25.68 & 91 \\
\hline $\mathrm{J} 2059+6247$ & 205909.56 & +624744.1 & G & 0.2670 & II & 4.7 & 1.15 & 20.262 & 107.7 & 3.6 & 25.35 & 37 \\
\hline $\mathrm{J} 2059+2434$ & 205939.81 & $\begin{array}{r}+243423.9 \\
\end{array}$ & G & $(0.116)$ & II & 8.2 & 1.06 & 17.740 & 149.0 & 8.0 & 24.70 & 4 \\
\hline J2230-3942 & 223040.28 & $-3942 \quad 52.1$ & $\mathrm{Q}$ & 0.3181 & II & 3.3 & 0.91 & & 591.1 & 17.7 & 26.26 & 2 \\
\hline $\mathrm{J} 2233+1315$ & 223301.30 & +131502.5 & G & $(0.093)$ & II & 16.0 & 1.71 & 16.504 & 125.0 & 4.0 & 24.42 & 4 \\
\hline J2234-0224 & 223458.76 & -022418.9 & Q & 0.5500 & II & 3.3 & 1.27 & 18.333 & 75.7 & 2.3 & 25.92 & 3 \\
\hline J2239-0133 & 223959.34 & -013351.4 & G & 0.0881 & II & 20.4 & 1.97 & 16.666 & 135.0 & 4.1 & 24.39 & 3 \\
\hline $\mathrm{J} 2242+6212$ & $22 \quad 4232.13$ & +621217.6 & G & 0.1880 & II & 4.2 & 0.78 & 21.896 & 288.7 & 8.8 & 25.44 & 37 \\
\hline J2245-0032 & 224520.76 & -003206.1 & G & $(0.66)$ & II & 3.0 & 1.25 & 22.018 & 17.0 & 0.6 & 25.46 & 23 \\
\hline $\mathrm{J} 2250+2844$ & 225039.16 & +284445.5 & G & (0.097) & II & 8.4 & 0.93 & 16.747 & 120.0 & 9.0 & 24.44 & 4 \\
\hline $\mathrm{J} 2253-3455$ & 225336.03 & -345530.8 & G & 0.2115 & II & 4.5 & 0.93 & & 291.3 & 8.7 & 25.56 & 2 \\
\hline J2253-5812 & 225358.98 & $-58 \quad 1249.4$ & G & 0.1764 & II & 4.3 & 0.76 & & 5.4 & 0.2 & 23.65 & 12 \\
\hline $\mathrm{J} 2256-3617$ & $2256 \quad 15.08$ & -361759.1 & $\mathrm{G}$ & 0.0902 & II & 14.5 & 1.51 & & 195.0 & 11.0 & 24.58 & 4 \\
\hline $\mathrm{J} 2257-0052$ & 225734.43 & -005231.8 & G & 0.5220 & II & 5.3 & 1.98 & 20.790 & 80.2 & 3.0 & 25.89 & 9 \\
\hline $\mathrm{J} 2326+2458$ & 232623.20 & +245840.4 & G & 0.2549 & II & 11.7 & 2.88 & 18.676 & 274.0 & 27.0 & 25.71 & 4 \\
\hline J2328-0825 & 232850.02 & $-08 \quad 2511.8$ & G & 0.3840 & II & 4.6 & 1.40 & 19.664 & 64.9 & 3.7 & 25.49 & 1,4 \\
\hline
\end{tabular}




\begin{tabular}{|c|c|c|c|c|c|c|c|c|c|c|c|c|}
\hline $\begin{array}{l}\text { IAU } \\
\text { name } \\
(1)\end{array}$ & $\begin{array}{c}\alpha(2000) \\
(\mathrm{h} \mathrm{m} \mathrm{s}) \\
(2)\end{array}$ & $\begin{array}{c}\delta(2000) \\
\left(\begin{array}{c}o \\
(3)\end{array}\right)\end{array}$ & (4) & (5) & $\begin{array}{c}\text { FR } \\
\text { type } \\
(6)\end{array}$ & $\begin{array}{c}\text { LAS } \\
(\operatorname{arcmin}) \\
(7)\end{array}$ & $\begin{array}{c}\mathrm{D} \\
(\mathrm{Mpc}) \\
(8)\end{array}$ & $\begin{array}{c}r \\
(\mathrm{mag}) \\
(9)\end{array}$ & $\begin{array}{c}\mathrm{S}_{I} \\
(\mathrm{mJy}) \\
(10)\end{array}$ & $\begin{array}{c}\delta S_{I} \\
(\mathrm{mJy}) \\
(11)\end{array}$ & $\begin{array}{c}\log \mathrm{P}_{t o t} \\
\mathrm{~W} / \mathrm{Hz} \\
(12)\end{array}$ & Ref. \\
\hline $\mathrm{J} 2333-2343$ & 233355.24 & -234340.7 & $\mathrm{G}$ & 0.0477 & II & 18.9 & 1.05 & 16.321 & 1167 & 35.1 & 24.78 & 2 \\
\hline $\mathrm{J} 2344-0032$ & 234440.04 & -003231.7 & $\mathrm{Q}$ & 0.5014 & II & 2.7 & 0.99 & 17.669 & 34.9 & 1.1 & 25.49 & 25 \\
\hline $\mathrm{J} 2345-0449$ & 234532.70 & -044925.4 & G & 0.0756 & II & 17.1 & 1.46 & 15.968 & 173.9 & 5.2 & 24.38 & 3 \\
\hline $\mathrm{J} 2355+7955$ & 235523.33 & +795519.6 & G & 1.3360 & II & 1.4 & 0.71 & & 1722 & 51.7 & 28.20 & 95 \\
\hline J2359-6054 & 235904.36 & -605459.3 & G & 0.0963 & II & 7 & 0.74 & & 26240 & 460 & 26.77 & 41 \\
\hline
\end{tabular}

Column description: (1) - source name, (2) and (3) - J2000.0 coordinates of the host optical object, (4) - optical identification (G - galaxy or Q - quasar), (5) redshift, (6) - radio morphological type, (7) - angular size in arcmin, (8) projected linear size in Mpc, (9) - optical aperture magnitude in $r$ band, (10) total flux-density at $1.4 \mathrm{GHz}$, unless indicated, in units of mJy, (11) - error of flux-density in mJy, (12) - total radio luminosity at $1.4 \mathrm{GHz}$ in units of W/Hz (14) - references.

Notes: (a) - flux-density at $1.388 \mathrm{GHz}$ from Saripalli et al. (2012), (b) - SUMSS measurement of flux-density, (c) - flux-density at 0.843 GHz from Saripalli et al. (2005), (d) - flux-density at $0.325 \mathrm{GHz}$ from Sebastian et al. (2018), (r) - optical $r$ magnitudes taken from Saripalli et al. (2012). The redshifts given in brackets are photometric n) - in Weżgowiec, Jamrozy \& Mack (2016) the diffuse radio emission can be seen. It extends for 23' and it is presumably the evidence of past radio emission, (m) GRG proposed by Mack et al. (2005). Its size is uncertain, (p) - we also found different value of photometric redshift equal to 0.473 (Brescia, Cavuoti \& Longo 2015 ).

References: 1. Bankowicz et al. (2015), 2. Proctor (2016), 3. Machalski, Kozieł-Wierzbowska \& Jamrozy (2007), 4. Dabhade et al. (2017), 5. Solovyov \& Verkhodanov (2014 ), 6. Kapahi et al. (1998), 7. Saripalli et al. (2012), 8. Subrahmanyan et al. (2010), 9. Sadler, Cannon \& Mauch (2007), 10. Saikia, Konar \& Kulkarni (2006), 11. Santiago-Bautista et al. (2016), 12. Saripalli et al. (2005), 13. Djorgovski et al. (1995), 14. Mack et al. (1997), 15. Andernach et al. (1992), 16. van Breugel \& Jägers (1982), 17. Laing, Riley \& Longair (1983), 18. Subrahmanyan, Saripalli \& Hunstead (1996), 19. Lacy (2000), 20. Colafrancesco et al. (2016), 21. Nilsson (1998), 22. Fomalont et al. (1978), 23. Rentería Macario \& Andernach (2017), 24. Sadler et al. (2002), 25. Kuźmicz \& Jamrozy (2012), 26. Schoenmakers et al. (2001), 27. Tamhane et al. (2015), 28. Simpson et al. (2006), 29. Koziel-Wierzbowska \& Stasińska (2011), 30. de Bruyn (1989), 31. Schoenmakers et al. (1998), 32. Saripalli, Subrahmanyan \& Hunstead (1994), 33. Hunik \& Jamrozy (2016), 34. Reid, Kronberg \& Perley (1999), 35. Hurley-Walker et al. (2015), 36. Amirkhanyan, Afanasiev \& Moiseev (2015), 37. Lara et al. (2001b), 38. Ishwara-Chandra \& Saikia (1999), 39. Filipovic et al. (2013), 40. Jägers (1986), 41. Malarecki et al. (2015), 42. Saripalli, Gopal-Krishna \& Kuehr (1986), 43. Kronberg, Wielebinski \& Graham (1986), 44. Cotter, Rawlings \& Saunders (1996), 45. Machalski, Jamrozy \& Zoła (2001), 46. Machalski et al. (2006), 47. Hes, de Vries \& Barthel (1995), 48. Kuligowska et al. (2009), 49. Lacy et al. (1993), 50. Coziol et al. (2017), 51. Sebastian et al. (2018), 52. Kuligowska et al. (2009), 53. Law-Green et al. (1995), 54. Proctor (2011), 55. Högbom (1979), 56. Machalski \& Condon (1999), 57. Owen et al. (2000), 58. Bhatnagar, Krishna \& Wisotzki (1998), 59. Kuźmicz, Kuligowska \& Jamrozy (2011), 60. Hintzen, Ulvestad \& Owen (1983), 61. van Breugel \& Willis (1981), 62. Saunders, Baldwin \& Warmer (1987), 63. Mack et al. (2005), 64. Schoenmakers et al. (2000a), 65. Machalski (1998), 66. Jones \& McAdam (1992), 67. Djorgovski et al. (1990), 68. Saripalli et al. (1997), 69. McCarthy et al. (1997), 70. Parma et al. (1996), 71. Gregg, Becker \& de Vries (2006), 72. Hota et al. (2011), 73. Machalski et al. (2008), 74. Falco et al. (1999), 75. Singal, Konar \& Saikia (2004), 76. Andernach et al. (2012), 77. Bassani et al. (2016), 78. Masetti, Parisi \& Palazzi (2013), 79. Molina et al. (2015), 80. Clarke et al. (2017), 81. Konar et al. (2006), 82. Baum \& Heckman (1989), 83. Röttgering et al. (1996), 84. Marecki, Jamrozy \& Machalski (2016), 85. Molina et al. (2014), 86. Lacy et al. (1999), 87. Lacy, Rawlings \& Warner (1992), 88. Lara et al. (1999), 89. Hagen, Engels \& Reimers (1999), 90. Saripalli et al. (2008), 91. Riley et al. (1989), 92. Jones et al. (2009), 93. Best et al. (2005), 94. Leahy \& Perley (1991), 95. Kharb, O’Dea \& Baum (2008). 